\title{
Correlation between Topographic N400 Anomalies and Reduced Cerebral Blood Flow in the Anterior Temporal Lobes of Patients with Dementia
}

\author{
Matthias Grieder ${ }^{\mathrm{a}, *}$, Raffaella M. Crinelli ${ }^{\mathrm{b}}$, Kay Jann ${ }^{\mathrm{a}, \mathrm{c}}$, Andrea Federspiel ${ }^{\mathrm{a}}$, Miranka Wirth ${ }^{\mathrm{d}}$, \\ Thomas Koenig $^{\mathrm{a}}$, Maria Stein ${ }^{\mathrm{a}}$, Lars-Olof Wahlund ${ }^{\mathrm{b}}$ and Thomas Dierks ${ }^{\mathrm{a}}$ \\ ${ }^{a}$ Department of Psychiatric Neurophysiology, University Hospital of Psychiatry, University of Bern, Switzerland \\ ${ }^{\mathrm{b}}$ Karolinska Institute, Department NVS, Division of Clinical Geriatrics, Stockholm, Sweden \\ ${ }^{\mathrm{c}}$ Department of Neurology, University of California Los Angeles, Los Angeles, CA, USA \\ ${ }^{\mathrm{d}}$ Helen Wills Neuroscience Institute, University of California, Berkeley, CA, USA
}

Handling Associate Editor: Claudio Babiloni

Accepted 11 April 2013

\begin{abstract}
In Alzheimer's disease (AD) patients, episodic memory impairments are apparent, yet semantic memory difficulties are also observed. While the episodic pathology has been thoroughly studied, the neurophysiological mechanisms of the semantic impairments remain obscure. Semantic dementia (SD) is characterized by isolated semantic memory deficits. The present study aimed to find an early marker of mild $\mathrm{AD}$ and SD by employing a semantic priming paradigm during electroencephalogram recordings. Event-related potentials (ERP) of early $(\mathrm{P} 1, \mathrm{~N} 1)$ and late $(\mathrm{N} 400)$ word processing stages were obtained to measure semantic memory functions. Separately, baseline cerebral blood flow (CBF) was acquired with arterial spin labeling. Thus, the analysis focused on linear regressions of CBF with ERP topographical similarity indices in order to find the brain structures that showed altered baseline functionality associated with deviant ERPs. All participant groups showed semantic priming in their reaction times. Furthermore, decreased CBF in the temporal lobes was associated with abnormal N400 topography. No significant $\mathrm{CBF}$ clusters were found for the early ERPs. Taken together, the neurophysiological results suggested that the automatic spread of activation during semantic word processing was preserved in mild dementia, while controlled access to the words was impaired. These findings suggested that N400-topography alterations might be a potential marker for the detection of early dementia. Such a marker could be beneficial for differential diagnosis due to its low cost and non-invasive application as well as its relationship with semantic memory dysfunctions that are closely associated to the cortical deterioration in regions crucial for semantic word processing.
\end{abstract}

Keywords: Alzheimer's disease, cerebral blood flow, event-related potential, magnetic resonance imaging, N400, semantic dementia, semantic memory, volumetry

\footnotetext{
${ }^{*}$ Correspondence to: Matthias Grieder, PhD, Department of Psychiatric Neurophysiology, University Hospital of Psychiatry, Bolligenstrasse 111, CH-3000 Bern 60, Switzerland. Tel.: +41 319328351; Fax: +41 319309961; E-mail: grieder@puk.unibe.ch.
}

\section{INTRODUCTION}

With the increasing population of elderly people, the prevalence of dementia increases from approximately $3 \%$ between 65 and 74 years to $25 \%-50 \%$ among those over 85 years [1,2]. Alzheimer's disease 
(AD), the most frequent type of dementia, is found in $70 \%$ of all patients affected [3]. Although the progressing deterioration of long-term memory, which affects episodic and semantic memory, is the major deficit in patients with $\mathrm{AD}$, additional cognitive abilities are disturbed, such as language and executive functions [4, 5]. However, these latter abilities have been shown to also be degraded in other dementia types, such as frontotemporal dementia (FTD). FTD is characterized by a spectrum of non-Alzheimer's dementias that mainly show frontal and/or temporal lobe degeneration [6]. The temporal variant of FTD has been described by a selective loss of semantic knowledge, and it is therefore referred to as semantic dementia (SD) [7, 8]. Alternatively, SD has been called the fluent variant of primary progressive aphasia [9].

Progress in neuroimaging techniques has recently allowed for the possibility of differential diagnosis of $\mathrm{AD}$ pathology by combining distinct biomarkers, such as the accumulation of amyloid- $\beta(A \beta)$, increased cerebral spinal fluid (CSF) tau, and neuronal atrophy [10]. However, $A \beta$ and tau markers can also be found in the elderly who do not develop the clinical syndrome of AD. In addition, the assessments of these biomarkers are costly and can be invasive for the patients [e.g., through the use of positron emission tomography (PET)]. Furthermore, neuronal atrophy, which appears to occur only shortly before the clinical manifestation of $\mathrm{AD}$, is rather difficult to distinguish from normal aging $[10,11]$. For SD, diagnostic criteria that are based primarily on behavioral measures have been developed [9, 12]. In summary, it appears that the use of only imaging methods, such as PET and magnetic resonance imaging (MRI), is less suited for the preventive screening of the potential development of dementia in the symptom-free elderly on a routine basis. Consequently, there is still a need for cognitive measures that distinctively detect the probable emergence of the clinical syndromes of AD and SD [13-15]. For instance, Jack et al. [10] have stated that the number of studies that combine biomarkers is limited. Hence, the current study aimed at finding a biomarker for the clinical syndrome of dementia that is based on underlying pathology by investigating measures that reflect the cognitive symptomatology and the neuronal degeneration. However, because the combination of the clinical syndrome and the pathology is difficult to assess in the pre-symptomatic stage, we examined patients who were in an early symptomatic stage of $\mathrm{AD}$ or SD. This study must therefore be considered a first step in the development of a cost-efficient and non-invasive biomarker of dementia, as any candidate marker needs to be tested in a longitudinal study beginning at the pre-symptomatic stage.

In the following, a theoretical background of the cognitive measures that target the clinical syndrome, as well as the neurophysiological methods that assess neuropathology, is provided. Generally, language function, communication, and every-day functioning rely strongly on an intact semantic memory. Even though semantic memory disturbances have been widely identified in patients with $\mathrm{AD}$ and can be measured with tasks, such as word fluency or object naming, the exact neurophysiological correlates of these disturbances remain unclear [16, 17]. Investigations of semantic memory dysfunctions with neurophysiological measures are thus needed to identify markers that can be used for the early clinical diagnosis of patients with AD. In particular, semantic memory functions are often examined through priming paradigms that are established research instruments that are utilized for the assessment of rather automatic semantic memory functions with minimized influence of explicit cognitive processes [18]. This is advantageous in order to avoid confounding factors, such as working memory or more general executive processes, especially because they co-occur as deficits in the dementia types. In particular, semantic priming is characterized as the facilitated retrieval of (target) words which are preceded by contextually related words, so-called primes. This effect is commonly detected by shorter reaction times (RTs) to related (e.g., forest - tree) than to unrelated (e.g., frog - guitar) word pairs. The resulting semantic priming effect has been attributed to the spread of neural activation within the semantic memory network [19].

Previous behavioral priming studies that have been conducted on patients with $\mathrm{AD}$ and SD have reported diverging results, including reduced [20], normal [21, 22], and increased priming [23], compared to healthy participants. Despite these inconsistencies, semantic priming can be regarded as a valuable tool for investigating the integrity of semantic memory functions if the paradigm is carefully designed and influencing factors (e.g., stimulus timing, word versus sentence task) are taken into account [24].

In addition to the behavioral domain, semantic priming effects have been examined by means of electrophysiological markers, such as event-related potentials (ERP). Wirth et al. [25] have demonstrated an automatic semantic priming effect in healthy participants that occurs between 120-190 ms after target-word onset (early ERP). During this period, the ERP components labeled as P1 and N1 can be observed, and these are possibly functionally linked 
with the automatic spread of activation in the semantic system. Furthermore, in numerous studies, the wellestablished controlled semantic priming effect has been found approximately between $250-500 \mathrm{~ms}$ (late ERP). This time frame coincides with the ERP component referred to as $\mathrm{N} 400$, thus reflecting a semantic integration stage [26, 27].

While investigating semantic processing in patients with $\mathrm{AD}$, the majority of studies have found reduced N400 amplitudes with delayed latencies compared to those in elderly controls (EC) [28]. Similarly, Schwartz et al. [29] have shown comparable N400 component amplitudes but smaller priming effects in patients with AD compared to those in young and elderly controls. Furthermore, their data for mixed auditory and visual stimuli with a rather long stimulus-onset asynchrony of approximately $1,000 \mathrm{~ms}$ have indicated that the priming effect occurred later in the patients with $\mathrm{AD}$ than in the healthy groups. However, they concluded that, despite the alterations in patients with $\mathrm{AD}$, their semantic network does not seem to be broken down severely, as shown by a normal N400 component amplitude. Similarly, Iragui and colleagues [30] have shown reduced and delayed N400 priming in patients with $\mathrm{AD}$ with a context-phrase task. According to those authors, the difference might have occurred due to altered attentional processes or weaker associative links that were related to more advanced age. Ford et al. [31] have found, while also assessing sentence processing in patients with $\mathrm{AD}$, reduced N400 priming effects in the semantic but not in the phonemic task and delayed latencies in both conditions. Although these results appeared to demonstrate a distinct semantic memory alteration in patients with $\mathrm{AD}$, the fact that their semantic condition involved a delayed-recognition test might raise doubts of whether their results were confounded by episodic memory processes. Similarly, Hurley and colleagues [32] have shown that altered N400 amplitudes are evoked by picture-picture and picture-word matching tasks in patients with the semantic variant of primary progressive aphasia compared to controls. The only study that has investigated the early ERP components (N1, P2) of semantic processing besides the late N400 in patients with AD so far has been the study conducted by Revonsuo and colleagues [33]. They found a comparable congruity effect between patients with AD and controls in P2. In contrast, this effect was smaller in the $\mathrm{N} 400$ in the AD group compared to the control group. Therefore, these authors have claimed that early lexical processes are preserved in patients with $\mathrm{AD}$, whereas semantic-conceptual stages are impaired. In accor- dance, Ford et al. [34] have found differing topographic N400 congruity effects in patients with AD compared to controls. Nevertheless, they demonstrated that the N400 priming effect and scalp distribution were stable across patients with $\mathrm{AD}$, regardless of whether they were able to name a picture correctly or not. This latter result has been replicated by Auchterlonie et al. [35]. Thus, these results have provided evidence that patients with AD have an impaired access to semantic concepts, although the knowledge remains intact. Moreover, Olichney et al. [36, 37] have shown that an abnormal N400 is associated with an increased risk for conversion from mild cognitive impairment to $\mathrm{AD}$ within three years.

In contrast to the studies that have shown altered ERPs in patients with $\mathrm{AD}$, there have been findings of equivalent N400 measures in patients with $\mathrm{AD}$. For example, Hamberger et al. [38] who also employed a sentence task found that the N400 was modulated to the same degree by semantic relation in probable AD patients and young controls. They assumed that the semantic deficits in patients with $\mathrm{AD}$ were expressed even at a later processing stage than the N400. Despite the considerable number of studies that have investigated semantic ERPs in patients with $\mathrm{AD}$, data on the early ERPs (i.e., P1/N1) of semantic priming that are induced by visual stimuli in patients with AD and SD are missing.

Although electrophysiological data represent a direct measure of brain activity, it is hard to draw conclusions from this data of the underlying structural changes and, thus, the pathology of AD and SD in comparison to controls. Commonly, brain atrophy in patients with $\mathrm{AD}$ and $\mathrm{SD}$ has been measured with structural MRI with voxel-based morphometry (VBM) [39-44].

Even though the well-circumscribed grey matter (GM) volume losses in these patients appear to have an important relationship with the patients' cognitive deficits, volumetric measures alone are not the optimal way to detect degenerative diseases in the pre-clinical phase, as stated above. In particular, comparisons of different biomarkers have been advised in order to validate their application [10]. One possibility could be an investigation of the functional integrity of the brains of early or pre-dementia patients with cerebral blood flow (CBF) measurements [45, 46]. More recently, MRI-based arterial spin labeling (ASL) has allowed assessments of the resting-state CBF in a non-invasive fashion in healthy participants and patients [14, 47].

In accordance with this advantage, the current study employed ASL in addition to a standard VBM analysis. 
The most prominent findings of the previous studies of patients with $\mathrm{AD}$ were reduced $\mathrm{CBF}$ in the inferior parietal lobe, the posterior cingulate gyrus, and the middle frontal gyrus, as well as the inferior temporal cortex, compared to controls [48, 49]. These results are in line with those of studies that have used the more classical, but invasive, PET method (e.g., [47]). However, hypermetabolism has been reported in the hippocampus and other medial temporal structures, as well as in the anterior cingulate gyrus [49-51]. Hypermetabolism that coincides with GM atrophy has been suggested to reflect compensatory neural activity, inflammation or the increased production of vasodilators [49]. No ASL studies have been conducted on patients with SD, but, a fluorodeoxyglucose PET study that was conducted by Diehl et al. [52] has revealed reduced glucose metabolism in the entire left temporal lobe and the right temporal pole in patients with SD.

Overall, despite the asynchronous onsets of the different biomarkers of dementia, the clinical syndrome of $\mathrm{AD}$ and $\mathrm{SD}$ appears to be strongly related to its pathology, as shown by the structural and functional aberrations that involve language-related areas among others in these patients.

In the present study, the above-circumscribed neurophysiological methods (i.e., ERP, VBM, and ASL) were applied in order to differentiate semantic memory dysfunctions in patients with $\mathrm{AD}$ and those with $\mathrm{SD}$ and relate them to the underlying resting CBF. In particular, this is the first study that has directly related ERPs of semantic processing to the individual CBF findings in early dementia patients. Hence, it aimed to identify a measure of deviant semantic word processing that reflects the symptomatology of the clinical syndromes of $\mathrm{AD}$ and $\mathrm{SD}$ and that is associated with brain regions showing altered blood flow.

The following hypotheses were made for the present study. First, in order to verify that the semantic paradigm conducted in this study actuated semantic processing, a robust semantic priming effect of RT was expected, at least for EC subjects and patients with AD. Second, the replication of the distinction of early and late ERPs in the semantic word processing of ECs was anticipated. Third, both GM volumes and regional CBF were hypothesized to be comparable to those found in previously published studies. In patients with $\mathrm{AD}$, medial temporal, parietal, and basal GM atrophies were expected, while, in patients with SD, the volume of the temporal pole and the adjacent lateral temporal gyri, in particular, were predicted to be decreased. Moreover, in patients with $\mathrm{AD}$, reduced $\mathrm{CBF}$ was expected in the parietal lobe, posterior cingulate gyrus, middle frontal gyrus, and inferior temporal cortex. In contrast, hypermetabolism was expected in the medial temporal areas. As outlined above for patients with $\mathrm{SD}$, hypometabolism was anticipated to converge with findings of GM atrophy, particularly in the anterior temporal lobe. Fourth, the following brain areas were expected to be involved in the combination of ERP and CBF: for the early ERPs, the occipital and posterior temporal lobes, and, for the late ERPs, the anterior temporal lobes [25, 53-55]. Due to the lack of previous studies on early ERPs in patients with AD and SD in general, it was hard to predict the outcome of the ERP analysis. Nevertheless, an altered N400 was expected in patients with $\mathrm{AD}$, as described above, and, if the patients with SD had a loss of semantic knowledge [40], all analyzed ERPs were predicted to be changed because both the spread of activation and word retrieval would have been disturbed.

\section{MATERIALS AND METHODS}

\section{Participants}

A total of 48 participants were examined (22 EC, $19 \mathrm{AD}, 7 \mathrm{SD})$. However, due to an inability to perform the task (4 ADs), not fulfilling the diagnostic criteria (1 AD, 2 SDs), excessive MRI artifacts (1 EC), technical problems (1 EC), or an incidental finding of a tumor (1 EC), 10 participants had to be excluded from the data analysis, thus resulting in a sample of 38 participants (19 EC, 14 AD, $5 \mathrm{SD}$ ). The reason for the small SD group was that recruitment was complicated due to its relatively rare prevalence, its difficult diagnosis, and the absence of cognitive abilities in the patients to understand the study procedure. All were native speakers of Swedish, and their vision was either normal or corrected to normal. The study complied with the Declaration of Helsinki and was approved by the Regional Ethics Committee of Stockholm, Sweden, and all participants provided written informed consents.

The participant recruitment was different for each group. While the ECs were recruited by advertisement, patients with AD were contacted while they were in treatment at the Memory Clinic of the Geriatric Department at Karolinska University Hospital in Huddinge, Sweden. Their diagnoses were made by expert clinicians and were in accordance with the ICD-10 criteria [56]. Patients with SD were recruited from all over Sweden in accordance with the criteria by Neary et al. [12]. As part of the standard clinical procedure, the patients underwent physical and psychiatric medical examinations, including standard blood analyses, 
structural neuroimaging examinations, lumbar puncture, as well as a neuropsychological assessment. Apart from the patients' AD or SD diagnosis, as well as concurrent medication, none of the participants was affected by any neurological or psychiatric disease or taking medication affecting the central nervous system. Furthermore, a resting electroencephalogram (EEG) was measured in order to rule out abnormal EEG patterns, such as signs of spikes and waves.

\section{Stimulus material and task}

The semantic priming paradigm employed in this study was adopted from Grieder and colleagues [24]. It involved a lexical decision (LD) task and was composed of stimulus pairs containing nouns and non-words. The stimulus material varied by two experimental factors (relatedness and concreteness) with two levels (i.e., unrelated [U], related $[R]$, concrete [C], or abstract [A]; Fig. 1B). This resulted in four experimental conditions of interest: UC, UA, RC, and RA. For the exact task construction and validation of the stimuli, please refer to Grieder et al. [24]. Generally, the paradigm consisted of 160 word (noun) pairs (40 per condition), 160 matched pronounceable non-word-noun and noun-non-word pairs and 32 filler word pairs (352 stimulus pairs altogether). The total number of stimulus pairs differed slightly from that in Grieder et al. [24] in order to keep the task duration as short as possible (approximately $21 \mathrm{~min}$ ), while the stimulus-onset asynchrony was prolonged (700 ms, Fig. 1A). In detail, stimulus pairs appeared on a computer screen in sequential order (white Arial bold font, size 28, on black background). At a distance of $90 \mathrm{~cm}$ from the screen, the visual angle of the stimuli ranged from $0.382^{\circ}$ to $0.637^{\circ}$ in height and $0.446^{\circ}$ to $4.263^{\circ}$ in width. After a red fixation cross appeared $(400 \mathrm{~ms})$, the prime or non-word appeared for $650 \mathrm{~ms}$. Next, an interstimulus interval of $50 \mathrm{~ms}$ was followed by the target or non-word, which was displayed until the participant responded or $1,500 \mathrm{~ms}$ at maximum. Succeeding the target, a white fixation cross appeared for another $1,500 \mathrm{~ms}$ to complete one stimulus trial. The participants were instructed to press one of two buttons on a button box immediately after the target appeared to indicate whether the stimulus pair contained a nonword at either the prime or target position. In particular, in cases in which the stimulus pair contained two words, the participants had to press the rightmost button with the index finger of their dominant (right) hand, whereas if a non-word appeared at either the prime or target position, a button press on the leftmost button was required with the index finger of the non-dominant (left) hand. The RT was determined as the elapsed time in $\mathrm{ms}$ between the target stimulus onset and the participant's button press. The task was presented with E-Prime software (version 1.2, Psychology Software Tools, Inc., Pittsburgh, PA, USA) which logged the RT online.

\section{Procedure}

The examinations of the participants were divided into two separate sessions; one involved the neuropsychological testing and the EEG, and the other involved the MRI measurement. The first session, which was
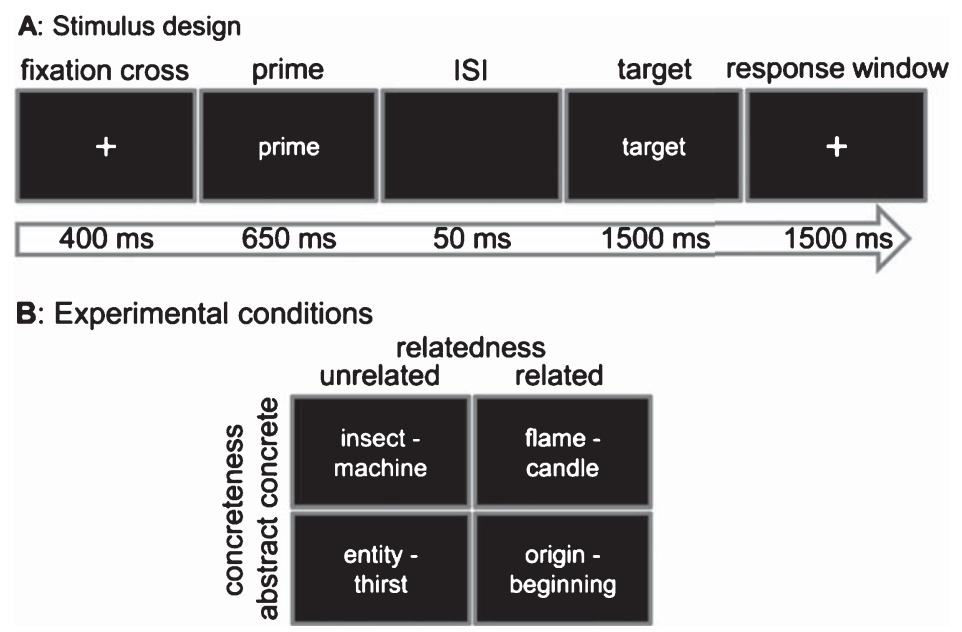

Fig. 1. A) Stimulus sequence of a trial with the corresponding screen duration (fixation cross in red; response window cross in white). B) The $2 \times 2$ factorial experimental design with word pair examples for each condition; filler and non-word conditions are not displayed. 
conducted at the phonetic laboratory of the Department of Linguistics at Stockholm University, started with an assessment of the participants' medical history. The subsequent neuropsychological examination included a vision screening, the Mini-Mental State Examination (MMSE), Boston Naming Test (BNT), Animal Fluency test (AF), Verb Fluency test (VF), Clock Task (CT, read and construct), and a computerized visuomotor RT task. Additionally, patients with $\mathrm{AD}$ and those with SD were tested with the Global Deterioration Scale (GDS) and the Cornell Depression Scale (CDS).

After the neuropsychological examination, the participants were seated on a chair in an electrically shielded room. As a next step, a resting EEG was measured for $6 \mathrm{~min}$ and $40 \mathrm{~s}$ with three periods of eyes closed ( 2 min each) and two periods of eyes opened (20 s each). Then, the LD task was introduced by means of a practice run. In particular, the participants read the task instructions on the screen and made LDs on 30 stimulus pairs (15 noun-noun and 15 noun-nonword pairs). The subsequent experimental LD task with simultaneous EEG/ERP recordings was only initiated if the participants were successful in the practice run.

The second session was performed at the Karolinska University Hospital in Huddinge, Sweden, where the MRI recordings were conducted. The participants were instructed to lie motionless in the scanner without falling asleep. In order to minimize motion artifacts, the participants' heads were carefully fixed by means of foam cushions. At first, a structural T1-weighted sequence was run. Second, a pseudo-continuous ASL (pCASL) measurement was conducted which concluded the data acquisition.

\section{Behavioral data analysis}

Possible group differences in demographics and neuropsychological tests were analyzed with nonparametric Kruskal-Wallis tests. Only the four word conditions, UC, UA, RC, and RA, were used for the analysis, and the filler and non-word conditions were not included. Offline, the median RT was calculated for each word condition and participant. Subsequently, a repeated-measures analysis of variance (ANOVA) with a $2 \times 2 \times 3$ factorial design (relatedness and concreteness as within-subject factors; group as between-subject factor) was conducted. Potential interactions were further disentangled by the Scheffé post-hoc test. The $d$-prime was calculated as a measure of individual task performance [57]. The resulting $z$-transformed scores were then subjected to a one-way ANOVA with a subsequent Scheffé post-hoc test.

\section{EEG/ERP recording and preprocessing}

Electrophysiological measurements were conducted with a high-impedance 128-channel HydroCel Geodesic Sensor Net connected to a Net Amps 300 amplifier (Electrical Geodesics, Inc., Eugene, OR, USA). A potassium-chloride solution was applied to the electrodes in order to keep the impedances below $50 \mathrm{k} \Omega$, and they were checked before the resting EEG and the ERP-EEG recordings. The recording reference was $\mathrm{Cz}$, and the ground electrode was positioned between $\mathrm{CPz}$ and $\mathrm{Pz}$. A fixed sampling rate of $20,000 \mathrm{~Hz}$ was low-pass filtered at $4,000 \mathrm{~Hz}$ and further down sampled online to $250 \mathrm{~Hz}$. Preprocessing was done with Vision Analyzer (Version 1.05, Brain Products GmbH, Gilching, Germany). An Independent Component Analysis [58] was computed to correct for eye movements and remove artifacts that were caused by eye blinks, repeatedly occurring electrode shifts, and/or cardio-ballistics. Channels containing muscle or other irregular artifacts were interpolated (order of splines $=4$; maximal degree of Legendre Polynomes $=10$; Lambda $=0.00001)$. Before the average reference was computed, the four channels located under each eye and ear were excluded from further analysis. The remaining epochs containing artifacts were removed by manual inspection. Moreover, an offline band-pass filter was applied at $0.5-18 \mathrm{~Hz}(24$ $\mathrm{db} / \mathrm{oct}$ ).

With the aim of extracting ERPs from the EEG, all experimental stimuli plus participant responses were marked online. Hence, segments starting from targetword onset and ending 1,000 ms after target-word onset were derived offline. Segments corresponding to false responses were rejected. Following this, the individual ERPs for the experimental conditions (UC, UA, RC, and RA) were averaged. Additionally, the individual average epoch over all conditions was derived, and, finally, the equivalent Global Field Power (GFP) [59] was calculated.

\section{ERP analysis}

The ERP analysis performed in this study mainly involved the two steps described in detail below. The aim was to obtain a suitable ERP measure that can be correlated with CBF. Therefore, the rationale was to find an appropriate measure reflecting the extent of deviation in semantic word processing in the healthy 
group ERPs. For this reason, a topographic approach appeared plausible because it accounted for the signals from all electrodes and identified neural activation modulations of the underlying source distribution [60]. As a consequence, the individual ERP topographies were spatially correlated with the mean ERP topography of the EC group as a measure of topographical similarity or deviation, respectively.

However, because the early ERP components (especially P1) are known to reflect visual perceptive processes, it was essential to verify that the P1 and $\mathrm{N} 1$ (as well as the N400) of the current study involved semantic processes by means of semantic priming, as demonstrated in Wirth et al. [25]. Therefore, a topographic ANOVA (TANOVA) that compared the individual averaged epochs of the unrelated and related word-pair conditions of the EC was conducted. The TANOVA is a non-parametric randomization test of reference-independent topographic differences [60]. In particular, 5,000 randomization runs were computed with a $p$-threshold of 0.05 (for details, see [24, 61, 62]).

\section{Peak detection}

For each ERP of interest (P1, N1 and N400), an automated peak detection that searched for local maxima was conducted with the Brain Vision Analyzer software. For this purpose, a pre-defined time window for each ERP was needed in order to avoid an overlap and subsequent confusion of the peaks to be detected. To this end, a separate topographic cluster analysis (microstates) [63] involving a $k$-means algorithm was performed on the mean epoch of the healthy participants with the Ragu software [61]. The resulting onsets and offsets of the microstates (corresponding to the P1, N1, and N400) were assigned as a time-frame limiter to the peak detection. In particular, the peaks were determined on the GFP and applied to all channels in order to extract the three peak topographies of each participant. Furthermore, the peak topographies were normalized $(\mathrm{GFP}=1)$ in order to remove electrical field strength differences from the topographies. Finally, all extracted topographies of the ECs were averaged in order to create the ERP topography template.

\section{Topographic component recognition}

Topographic component recognition (TCR) [64] was employed in order to obtain a parameter of the individual topographic similarities (i.e., spatial correlation) of the ERPs to the topography templates of the ECs. The individual ERP topographies that were derived from the peak detections were simply correlated with the group topography of the ECs (i.e., template map) with a self-written MATLAB script. The outcome variable of the TCR was one $r$ value per ERP and participant.

The advantage of the TCR compared to conventional single-channels or channel-groups analyses shall be briefly outlined. The TCR analysis extracts the strength of a component as a weighted mean of all electrodes, whereas the weights are given by the template map. An analysis of single channels or group channels is a very similar procedure, with the only difference being that the weights of the included electrodes are set to 1 , and the weights of the excluded electrodes are 0 . Single-channel and group-channel analyses are thus special cases of topographic analyses, with predefined binary template maps. If a template map that covers the whole scalp is given, as was the case in this study, a reduction to the binary form would possibly reduce the statistical power of the analyses. In other words, if only a few electrodes accounted for the effect of interest, the template map would give all the weights to those electrodes, which is very similar to the use of just those few electrodes.

\section{MRI recording}

A 3T Siemens Magnetom Trio MR Scanner (Siemens AG, Erlangen, Germany) was used for MRI data acquisition. The parameters of the T1-weighted magnetization-prepared rapid acquisition gradientecho (MPRAGE) sequence were set as follows: repetition time (TR)/echo time (TE), 1,900 ms/2.57 ms; 176 sagittal slices; slice thickness, $1.0 \mathrm{~mm}$; field of view (FOV), $230 \times 230 \mathrm{~mm}$; matrix size, $256 \times 256$; leading to a voxel dimension of $0.9 \times 0.9 \times 1.0 \mathrm{~mm}$. The pCASL measurement was applied $[65,66]$ with these parameters: TR/TE/post-label delay $(\omega) /$ tagging duration $(\tau)[\mathrm{ms}], 3500 / 18 / 1170 / 1600 ; 18$ horizontal slices; FOV, $230 \times 230$; matrix size, $64 \times 64$ and voxel size, $3.6 \times 3.6 \times 6.0 \mathrm{~mm}$; gap between slices, $0.9 \mathrm{~mm}$; slice acquisition time, $45 \mathrm{~ms}$ and TA, $8 \mathrm{~min} 22 \mathrm{~s}$.

\section{$V B M$}

Processing of the structural images from the MPRAGE sequences was performed with statistical parametric mapping software (SPM8, Wellcome Laboratory of Imaging Neuroscience, London, England; http://www.fil.ion.ucl.ac.uk) in order to test for regional differences in GM volume between the 
participant groups. Therefore, for the VBM analysis [67], the optimized protocol described in Good et al. [68] was applied.

Thus, the following computation steps were conducted. First, the structural images were automatically segmented into GM, white matter (WM) and CSF. Next, spatial normalization was applied [leading to Montreal Neurological Institute (MNI) normalized images], which was followed by the modulation option that was chosen for this study in order to preserve the volume of each tissue within a voxel [68]. Finally, the segmented, normalized, and modulated images were smoothed with an isotopic Gaussian kernel of $10 \mathrm{~mm}$ Full Width at Half Maximum (FWHM). Additionally, the total intracranial volume (TIV) was derived from the addition of the GM, WM, and CSF volumes of each participant, and it was used as a covariate in the statistical VBM analysis.

\section{CBF quantification}

The pCASL images were preprocessed by realignment correction for motion artifacts, coregistration to the individual structural images, and normalization into MNI space. While the preprocessing steps were done with SPM8 routines, the quantification was computed with in-house MATLAB scripts (Version 7.6; The MathWorks Inc., Natick, MA, USA). The individual regional $\mathrm{CBF}$ values were computed with the following quantification equation:

$$
\begin{aligned}
C B F= & \left(\frac{\lambda \cdot \Delta M}{2 \cdot \alpha \cdot M_{0} \cdot T_{1 b}}\right) \cdot \\
& \left(\frac{1}{e^{-w / T_{1 b}}-e^{-(\tau+w) / T_{1 b}}}\right)
\end{aligned}
$$

According to Wu et al. [65], the blood/tissue water partition coefficient $(\lambda)$ was fixed at $0.9[\mathrm{~g} / \mathrm{ml}]$, the tagging efficiency $(\alpha)$ at 0.95 and the decay time for labeled blood $\left(\mathrm{T}_{\mathrm{lb}}\right)$ for $3.0 \mathrm{~T}$ magnetic fields at $1,490 \mathrm{~ms} . \mathrm{M}_{0}$ was the equilibrium brain tissue magnetization images $[69,70] . \Delta \mathrm{M}$ was obtained by simply subtracting the label from the control images. The derived difference was proportional to the CBF [71]. Subsequently, the temporal average across all volumes was calculated and spatial smoothing with a Gaussian kernel $(\mathrm{FWHM}=8 \mathrm{~mm}$ ) was applied to the resulting $\mathrm{CBF}$ images in order to increase the signal-to-noise ratio [72].

\section{Voxel-based statistics}

For the VBM analysis, modulated GM images were employed in a voxel-by-voxel fashion for group comparisons with an analysis of covariance (ANCOVA), with TIV as the covariate. The statistical approach was similar to that of Focke et al. [73] with a threshold of $p<0.0001$ (uncorrected) applied to the $F$-statistics. The resulting clusters were corrected for multiple comparisons with a family-wise error rate (FWE) of $p<0.05$. Post-hoc $t$-tests were conducted in order to inspect the GM volume differences (Bonferroni corrected $p$-threshold) between $\mathrm{AD}$ versus $\mathrm{EC}, \mathrm{SD}$ versus EC and AD versus SD.

For the voxel-based analysis of $\mathrm{CBF}$, individual GM masks were created in order to conduct the CBF statistics that were based on GM-corresponding voxels only. The resulting masked $\mathrm{CBF}$ images were subjected to an ANCOVA with the global GM CBF as a covariate. Equivalent to the VBM analysis, $F$ and $t$ statistics were evaluated in order to obtain significant group differences in $\mathrm{CBF}$.

Finally, for each ERP of interest (P1/N1/N400), a voxel-based linear regression was employed with GMmasked CBF images and individual $r$ values of the TCR as the regressor [74]. Global CBF was included as a covariate.

\section{Correlations of the physiological parameters with the behavioral variables}

In order to investigate the relationship of the neuropsychological test scores with the $\mathrm{CBF}$ and ERP variables, a Spearman rank correlation was conducted.

\section{RESULTS}

\section{Behavioral data}

The demographics, neuropsychological scores, and statistics are listed in Table 1. Neither age nor education was significantly different between the groups. Therefore, these variables were not used as covariates. Second, the neuropsychological tests demonstrated the following selective deficits in the patient groups: while the MMSE, BNT, AF, and VF scores were reduced in patients with $\mathrm{AD}$ and those with $\mathrm{SD}$, they performed as well as the EC in the CT tasks. Note that the groupwise post-hoc Mann-Whitney tests showed significant differences in the MMSE, BNT, AF, and VF scores between $\mathrm{EC}$ and $\mathrm{AD}, \mathrm{EC}$ and $\mathrm{SD}$ as well as $\mathrm{AD}$ and SD at $p<0.01$, except for the MMSE and VF scores 
Table 1

Descriptive statistics and analysis of the demographics and neuropsychological tests

\begin{tabular}{|c|c|c|c|c|c|c|c|c|c|}
\hline & \multicolumn{2}{|c|}{$\mathrm{EC}$} & \multicolumn{2}{|c|}{$\mathrm{AD}$} & \multicolumn{2}{|c|}{ SD } & \multicolumn{3}{|c|}{ Analysis } \\
\hline & Mean & $S D$ & Mean & $S D$ & Mean & $S D$ & $x^{2}$ & $d f$ & $p$ \\
\hline \multicolumn{10}{|c|}{ Demographics } \\
\hline Age & 69.5 & 3.1 & 66.5 & 9.6 & 65.8 & 3.8 & 3.59 & 2 & 0.17 \\
\hline Education & 13.9 & 3.0 & 13.8 & 4.2 & 13.6 & 2.9 & 0.19 & 2 & 0.91 \\
\hline \multicolumn{10}{|c|}{ Neuropsychological tests } \\
\hline MMSE & 28.7 & 0.9 & 24.8 & 3.9 & 23.0 & 5.4 & 21.20 & 2 & $<0.001 * * *$ \\
\hline BNT & 54.0 & 3.9 & 46.3 & 6.1 & 9.4 & 7.4 & 22.73 & 2 & $<0.001 * * *$ \\
\hline $\mathrm{AF}$ & 23.8 & 6.3 & 14.9 & 2.2 & 5.6 & 4.3 & 23.41 & 2 & $<0.001 * * *$ \\
\hline VF & 21.6 & 5.8 & 12.4 & 4.3 & 9.0 & 6.6 & 19.34 & 2 & $<0.001 * * *$ \\
\hline CT construct & 3.8 & 1.4 & 3.6 & 0.8 & 3.3 & 2.0 & 1.25 & 2 & 0.54 \\
\hline CT read & 4.6 & 0.9 & 4.3 & 1.2 & 4.8 & 0.4 & 1.07 & 2 & 0.59 \\
\hline
\end{tabular}

EC, elderly controls; AD, Alzheimer's disease; SD, semantic dementia; SD, standard deviation; MMSE, Mini-Mental State Examination; BNT, Boston Naming Test; AF, animal fluency; VF, verb fluency; CT, clock task.

which did not differ significantly between the AD and SD groups.

Figure 2 depicts the descriptive group RTs per word condition, and Table 2 shows the ANOVA results. Generally, all groups showed semantic priming (i.e., relatedness effect, shorter RT for related words compared to RTs for unrelated words). Consequently, these results indicated that the participants processed the stimulus material semantically and not only lexically, as intended. This finding is important for the correlations of the task-related ERPs and the CBF in brain structures that are associated with semantic processing. Note that the concreteness effect was not of interest in this study. More importantly, a group main effect together with the post-hoc test showed that the SD group exhibited longer RTs than the EC and AD groups. For accuracy, the one-way ANOVA revealed a significant group effect of $d$-prime scores (Table 2). The Scheffé post-hoc test confirmed that all groups differed significantly from each other in task performance, with the EC group performing best and the $\mathrm{SD}$ group performing worst $(\mathrm{EC}-\mathrm{AD}=0.87, S E=0.22$, $p<0.01 ; \quad \mathrm{EC}-\mathrm{SD}=1.90, \quad S E=0.32, p<0.001 ; \mathrm{AD}-$ $\mathrm{SD}=1.03, S E=0.33, p<0.05)$.

\section{ERP}

Figure 3 depicts a first approach to the ERP data analysis that shows example electrode waveforms (F3, $\mathrm{T} 6$, and $\mathrm{Pz}$ ) between the unrelated and related word conditions for each participant group. Additionally, the GFP waveform illustrates the electrical field strength of all electrodes over time. What can be drawn from these waveforms as well as from the topographic map series (Fig. 4A) is that the early P1 and N1 components occurred in all three groups, although the amplitudes might have been reduced in patients with SD (see for
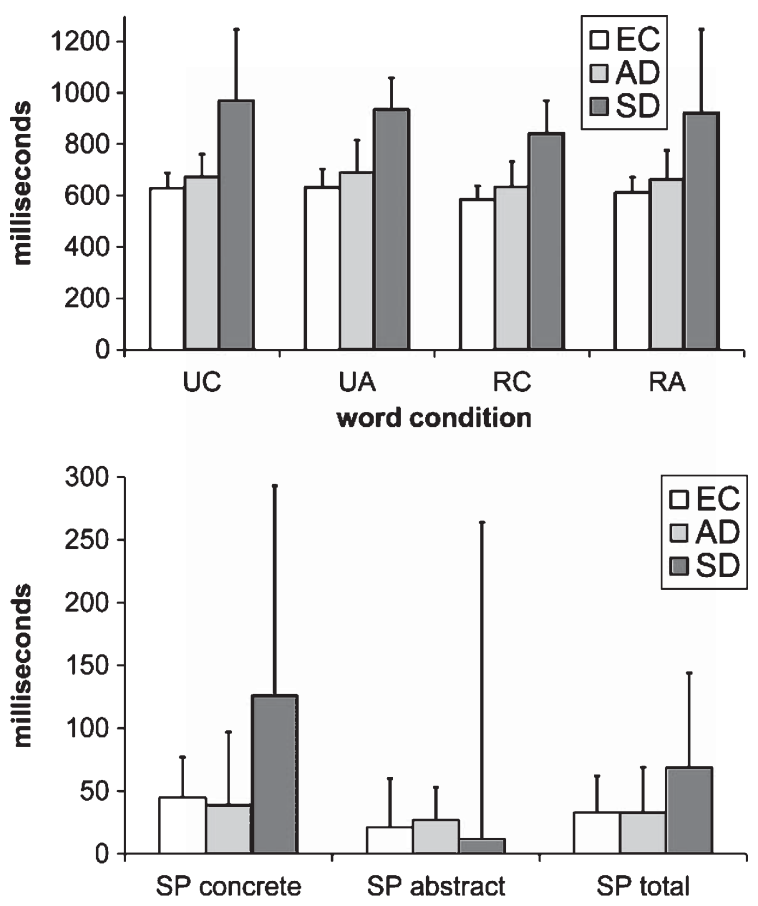

Fig. 2. Upper panel: Bar graph showing the mean reaction times (RTs) of all word conditions per participant group. The bars represent standard deviations. Lower panel: Mean semantic priming (SP) effects for concrete, abstract, and total of each group. UC; unrelated concrete, UA; unrelated abstract, RC; related concrete, RA; related abstract, EC; elderly controls, AD; Alzheimer's disease, SD; semantic dementia.

example electrode T6 in Fig. 3). Moreover, amplitude differences can be observed between the unrelated and related word condition in the $\mathrm{P} 1$ and $\mathrm{N} 1$ components, especially pronounced in the N1. Also, the waveforms indicate that the amplitude modulation between unrelated and related word pairs in the time window of the N400 component was strongest in the healthy controls 
Table 2

Statistical analyses of reaction time, accuracy, and topographic component recognition

\begin{tabular}{|c|c|c|c|c|c|c|c|c|c|}
\hline \multicolumn{10}{|l|}{ Reaction times } \\
\hline ANOVA & & & & & & & $F$ & $d f$ & $p$ \\
\hline Relatedness & & & & & & & 35.936 & 1 & $<0.001 * * *$ \\
\hline Concreteness & & & & & & & 5.961 & 1 & $<0.05^{*}$ \\
\hline Relatedness $\times$ group & & & & & & & 1.805 & 2 & 0.18 \\
\hline Concreteness $\times$ group & & & & & & & 0.173 & 2 & 0.84 \\
\hline Relatedness $\times$ concreteness & & & & & & & 3.480 & 1 & 0.07 \\
\hline Relatedness $\times$ Concreteness $\times$ group & & & & & & & 1.003 & 2 & 0.38 \\
\hline Group & & & & & & & 18.180 & 2 & $<0.001 * * *$ \\
\hline Post-Hoc Test (Scheffé) & & & & & & & Mean difference & $S E$ & $p$ \\
\hline $\mathrm{EC}-\mathrm{AD}$ & & & & & & & -51.9 & 35.3 & 0.35 \\
\hline $\mathrm{EC}-\mathrm{SD}$ & & & & & & & -303.4 & 50.4 & $<0.001 * * *$ \\
\hline $\mathrm{AD}-\mathrm{SD}$ & & & & & & & -251.5 & 52.2 & $<0.001 * * *$ \\
\hline \multicolumn{10}{|l|}{ Accuracy } \\
\hline & \multicolumn{2}{|c|}{$\mathrm{EC}$} & \multicolumn{2}{|c|}{$\mathrm{AD}$} & \multicolumn{2}{|c|}{ SD } & \multicolumn{3}{|c|}{ Analysis } \\
\hline & Mean & $S D$ & Mean & $S D$ & Mean & $S D$ & $F$ & $d f$ & $p$ \\
\hline Hit rate & 0.98 & 0.01 & 0.95 & 0.06 & 0.93 & 0.06 & 3.738 & 2 & $<0.05^{*}$ \\
\hline False alarm rate & 0.05 & 0.06 & 0.11 & 0.05 & 0.38 & 0.29 & 17.495 & 2 & $<0.001 * * *$ \\
\hline$d$ '-value & 3.98 & 0.54 & 3.11 & 0.47 & 2.07 & 1.23 & 20.239 & 2 & $<0.001 * * *$ \\
\hline \multicolumn{10}{|l|}{ Topographic component recognition } \\
\hline & \multicolumn{2}{|c|}{$\mathrm{EC}$} & \multicolumn{2}{|c|}{$\mathrm{AD}$} & \multicolumn{2}{|c|}{ SD } & \multicolumn{3}{|c|}{ Analysis } \\
\hline & Mean & $\overline{S D}$ & Mean & $S D$ & Mean & $S D$ & $\chi^{2}$ & $d f$ & $p$ \\
\hline $\mathrm{P} 1$ & 0.76 & 0.19 & 0.66 & 0.30 & 0.54 & 0.23 & 3.599 & 2 & 0.17 \\
\hline N1 & 0.62 & 0.29 & 0.41 & 0.42 & 0.34 & 0.40 & 4.868 & 2 & 0.09 \\
\hline N400 & 0.61 & 0.27 & 0.25 & 0.34 & -0.01 & 0.22 & 14.43 & 2 & $<0.01 * *$ \\
\hline
\end{tabular}

EC, elderly controls; AD, Alzheimer's disease; SD, semantic dementia; $S D$, standard deviation.

and attenuated in patients with AD. For patients with $\mathrm{SD}$, the $\mathrm{N} 400$ can be hardly identified in the waveforms or the topographic maps. The GFP of the AD group appeared to be comparable with that of the healthy controls in the P1 time window, but seemed to be decreased in the later ERP components. For patients with SD, the GFP seems to be reduced especially in the unrelated word pair condition during the $\mathrm{P} 1$ and $\mathrm{N} 1$ components, while the image is more diffuse in later time windows.

In the statistical ERP analysis, Fig. 4B illustrates that the TANOVA detected the early semantic priming effect that was measured by the topographical differences between $94 \mathrm{~ms}$ and $302 \mathrm{~ms}$ after target onset. Furthermore, there was a late topographical semantic priming effect between $382 \mathrm{~ms}$ and $546 \mathrm{~ms}$. Thus, all ERPs of interest (P1, N1, and N400) reflected semantic processing, as demonstrated by the overlap of the significant TANOVA epochs with the ERPs marked in Fig. 4C. In detail, Fig. 4C displays the temporal assignment of the eight microstates that were provided by the $k$-means cluster analysis overlaid with the GFP of the mean epoch of the EC group. Furthermore, the P1, N1, and N400 are labeled, as well as their corresponding time frame of occurrence (i.e., onset and offset). Note that, for the P1, the first GFP trough at $56 \mathrm{~ms}$ was chosen as the onset, instead of the $0 \mathrm{~ms}$ resulting from the clustering algorithm, which did not appear to be appropriate for the P1. This view was supported by the fact that the early TANOVA effect did not start before $94 \mathrm{~ms}$ and that no studies have reported semantic processing before $50 \mathrm{~ms}$ after stimulus onset [25, 53, 75]. Additionally, the group maps (templates for the EC group) of the P1, N1, and N400 of each group are depicted as topographical maps in Fig. 4D. Furthermore, as can be derived from Table 2, the Kruskal-Wallis test of the TCR resulted in significantly different correlations between the groups in the N400 only. The subsequent Scheffé post-hoc test showed that this effect was caused by differences between the EC and $\mathrm{AD}$ groups as well as between the $\mathrm{EC}$ and $\mathrm{SD}$ groups $(\mathrm{EC}-\mathrm{AD}=0.36$, $S E=0.10, p<0.01 ; \mathrm{EC}-\mathrm{SD}=0.62, S E=0.15, p<0.01$; $\mathrm{AD}-\mathrm{SD}=0.26, S E=0.15, p=0.25)$.

\section{$V B M$}

The participant groups did not differ in total GM volume as shown by the Kruskal-Wallis test $\left[\chi^{2}(2\right.$, $38)=2.05, p=0.36]$. However, the voxel-based statistics resulted in five significant clusters of differing 


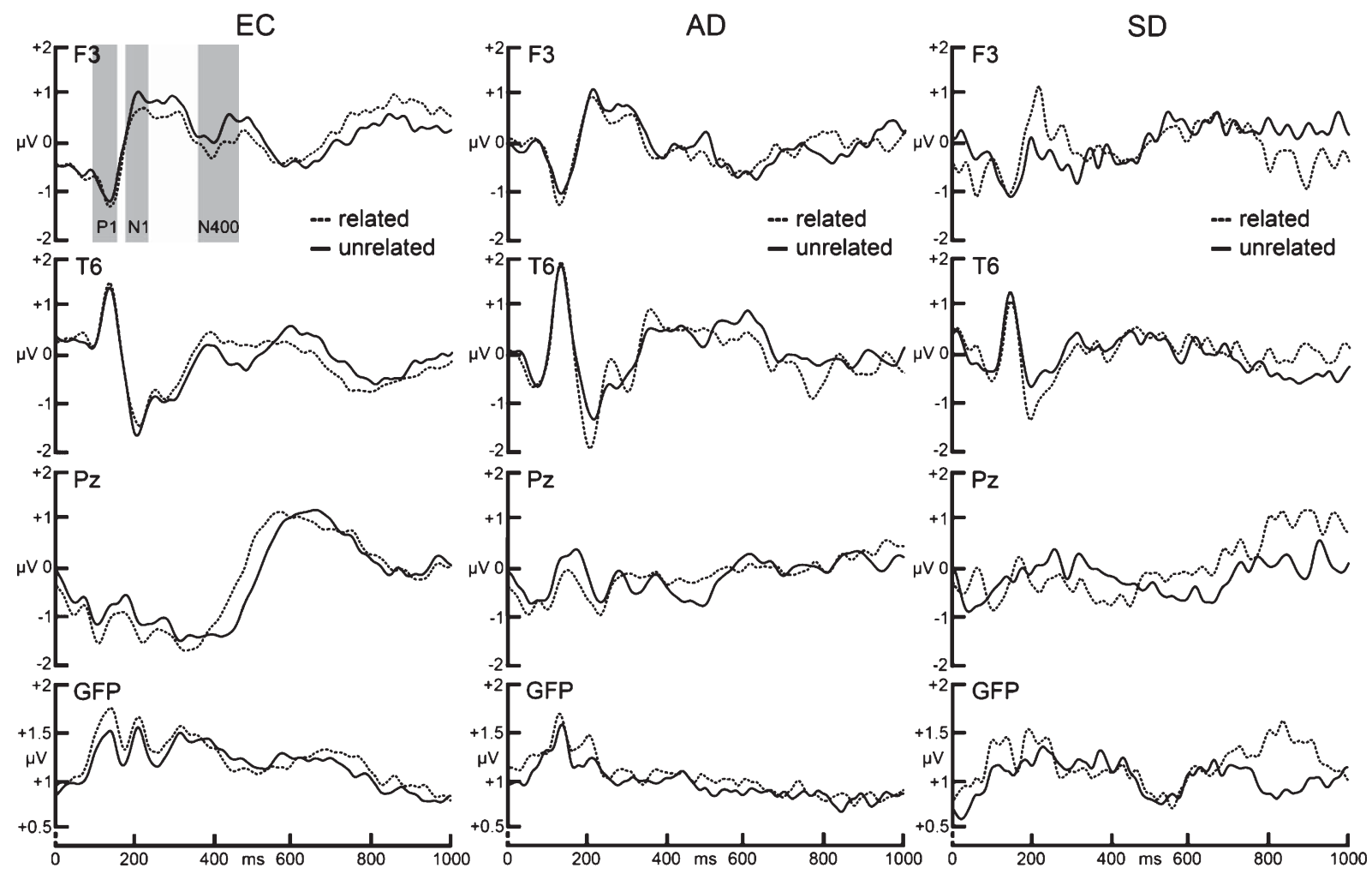

Fig. 3. Traditional waveform graph of three selected electrodes computed against the common average reference and the Global Field Power (GFP) of all electrodes. The mean event-related potentials (ERPs) of unrelated and related word pair conditions for each participant group are displayed as a function of time after target-word onset. The ERP components of interest (P1, N1, and N400) are marked with a grey bar in the top left panel. EC; elderly controls, AD; Alzheimer's disease, SD; semantic dementia.

GM volumes (Table 3). As shown in Fig. 5A (visualized with xjView toolbox, http://www.alivelearn. net/xjview), the largest cluster ( $F$-peak at $X=-30$, $\mathrm{Y}=-6, \mathrm{Z}=-34 ; \mathrm{MNI})$ extended from the left fusiform gyrus over the parahippocampal, hippocampal, and inferior temporal gyri and the temporal pole to the insula. Furthermore, even the left putamen and amygdala were involved. Additionally, in order to investigate the possible regional GM volume differences between the groups within this cluster, a voxel-wise $T$-test was conducted. As expected, between the EC and AD groups, the GM volume differed in the hippocampus, parahippocampal area, amygdala, and inferior temporal lobe (Fig. 5B). Furthermore, patients with SD showed reduced GM volume in the entire cluster compared to the other two groups. In the whole-brain VBM analysis, a comparable cluster can be observed in the right hemisphere, but to a smaller voxel extent ( $F$ peak at $\mathrm{X}=42$, $\mathrm{Y}=14, \mathrm{Z}=-30$ ). In addition, the SD group exhibited enhanced GM volume in the left inferior parietal lobule compared to the AD group. Next, the SD group exhibited increased GM volume in the right middle occipital gyrus compared to the other groups. Finally, the $\mathrm{AD}$ group had a lower GM volume in the right middle frontal gyrus compared to the EC group.

\section{$C B F$}

The AD group had lower global GM CBF compared to the EC group, as revealed by the Scheffé post-hoc test $\left(\mathrm{EC}-\mathrm{AD}=11.19, \mathrm{SE}=2.34, p<0.001^{* * *}\right)$ that was preceded by the Kruskal-Wallis test $\left[\chi^{2}(2,38)=13.65\right.$, $\left.p<0.01^{* *}\right]$. As in the VBM analysis, the largest cluster that was found with the CBF statistics extended further than the $F$ peak $(\mathrm{X}=-54, \mathrm{Y}=6, \mathrm{Z}=-32)$ only would indicate (Table 4). Instead, this cluster ranged from the left temporal pole over the fusiform gyrus to the hippocampus and the parahippocampal region (Fig. 5C). For this cluster, the $t$-tests demonstrated significantly decreased $\mathrm{CBF}$ in the $\mathrm{AD}$ and $\mathrm{SD}$ groups compared to the EC group. Similarly, reduced CBF was also observed in the left inferior temporal gyrus in both patient groups. Moreover, they exhibited 


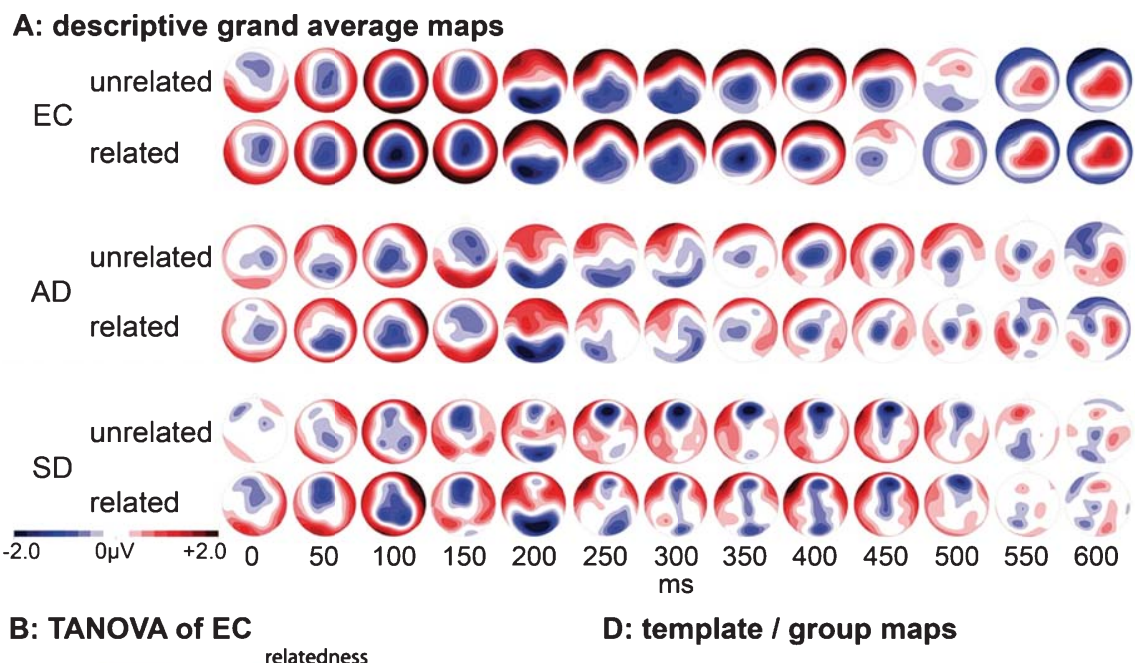

\section{B: TANOVA of EC}
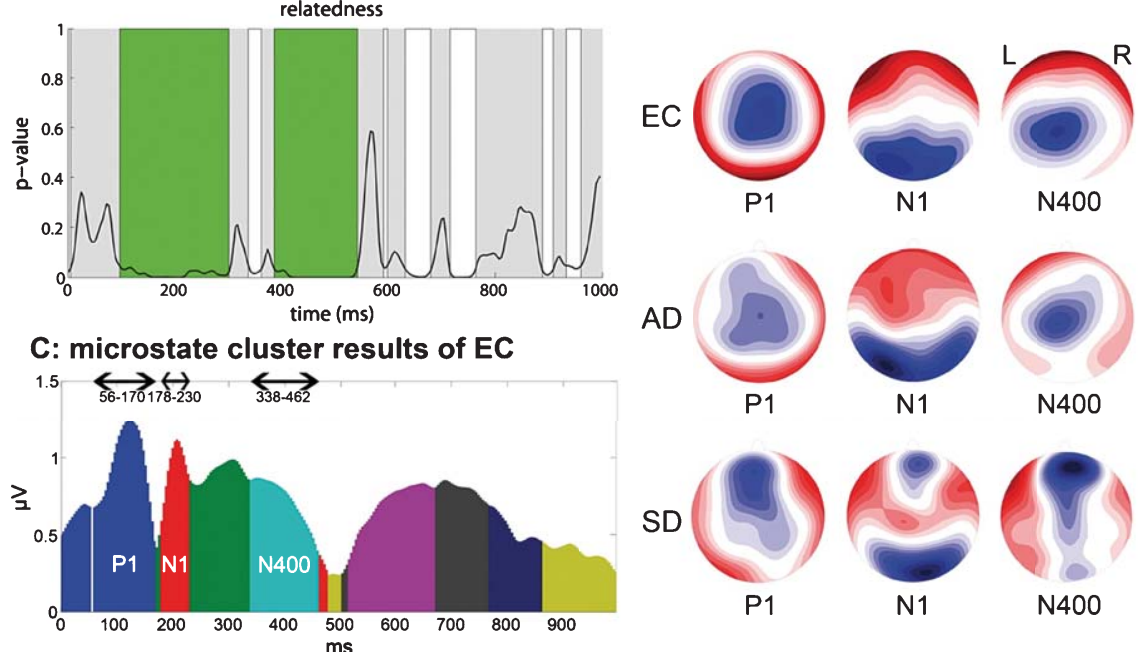

$A D$

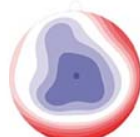

P1
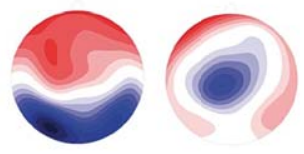

N1

N400
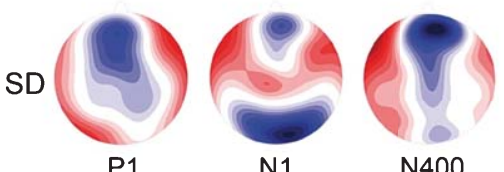

Fig. 4. A) Descriptive topographic grand average maps of unrelated and related word conditions for each participant group are depicted. B) Topographical relatedness (priming) effect of the topographic analysis of variance (TANOVA) in the elderly control (EC) group. The grey areas indicate non-significant topographic differences. The white areas show time epochs of the topographic differences without fulfilling the statistical criterion of minimal duration (i.e., were shorter than 0.95 of all resulting effect durations). Hence, the white areas could have occurred by chance only. The green areas reflect significantly differing topographies between unrelated and related word pairs. C) Microstate cluster analysis results in the 1,000-ms epoch after target-word onset overlaid with the mean Global Field Power (GFP) of the EC group. D) The group event-related potentials depicted as a topographical map. The maps of the EC group served exclusively as templates for the topographic component recognition. Remark: The low amplitudes are due to GFP normalization. AD; Alzheimer's disease, SD; semantic dementia.

Table 3

Voxel-based-morphometry analysis of the anatomical regions that correspond to the Montreal Neurological Institute (MNI)-coordinates at the $F$ peak, the number of involved voxels and the peak $F$ - and $p_{F W E-c o r r}$ values. Therefore, other brain structures were involved in the clusters, especially in the large ones (see text). Additionally, the post-hoc $T$-test results are listed with $T$ and $p$ values

\begin{tabular}{lrrrcllll}
\hline Anatomical region & \multicolumn{1}{c}{$\mathrm{X}$} & \multicolumn{1}{c}{$\mathrm{Y}$} & \multicolumn{1}{c}{$\mathrm{Z}$} & \# of voxels & $F\left(p_{F W E-c o r r}\right)$ & \multicolumn{1}{c}{ EC-AD $T(p)$} & EC-SD $T(p)$ & AD-SD $T(p)$ \\
\hline L fusiform gyrus & -30 & -6 & -34 & 7180 & $63.24\left(<0.001^{* *}\right)$ & $2.56(0.016)$ & $7.58\left(<0.001^{* *}\right)$ & $5.87\left(<0.001^{* *}\right)$ \\
R middle temporal pole & 42 & 14 & -30 & 4602 & $30.59\left(<0.01^{* *}\right)$ & $2.70(0.011)$ & $5.59\left(<0.001^{* *}\right)$ & $3.77\left(<0.01^{*}\right)$ \\
R middle occipital gyrus & 18 & -94 & 12 & 98 & $28.18\left(<0.05^{*}\right)$ & $0.23(0.82)$ & $-4.15\left(<0.01^{*}\right)$ & $-4.04\left(<0.01^{*}\right)$ \\
L inferior parietal lobule & -48 & -56 & 42 & 143 & $26.12\left(<0.05^{*}\right)$ & $2.65(0.013)$ & $-2.44(0.04)$ & $-4.49\left(<0.01^{*}\right)$ \\
R middle frontal gyrus & 32 & 36 & 30 & 95 & $24.18\left(<0.05^{*}\right)$ & $4.16\left(<0.001^{* *}\right)$ & $-0.71(0.5)$ & $-2.32(0.07)$ \\
\hline
\end{tabular}

$T$-test crit. $p$-value: $p<0.01 ; \mathrm{L}$, left; $\mathrm{R}$, right. 


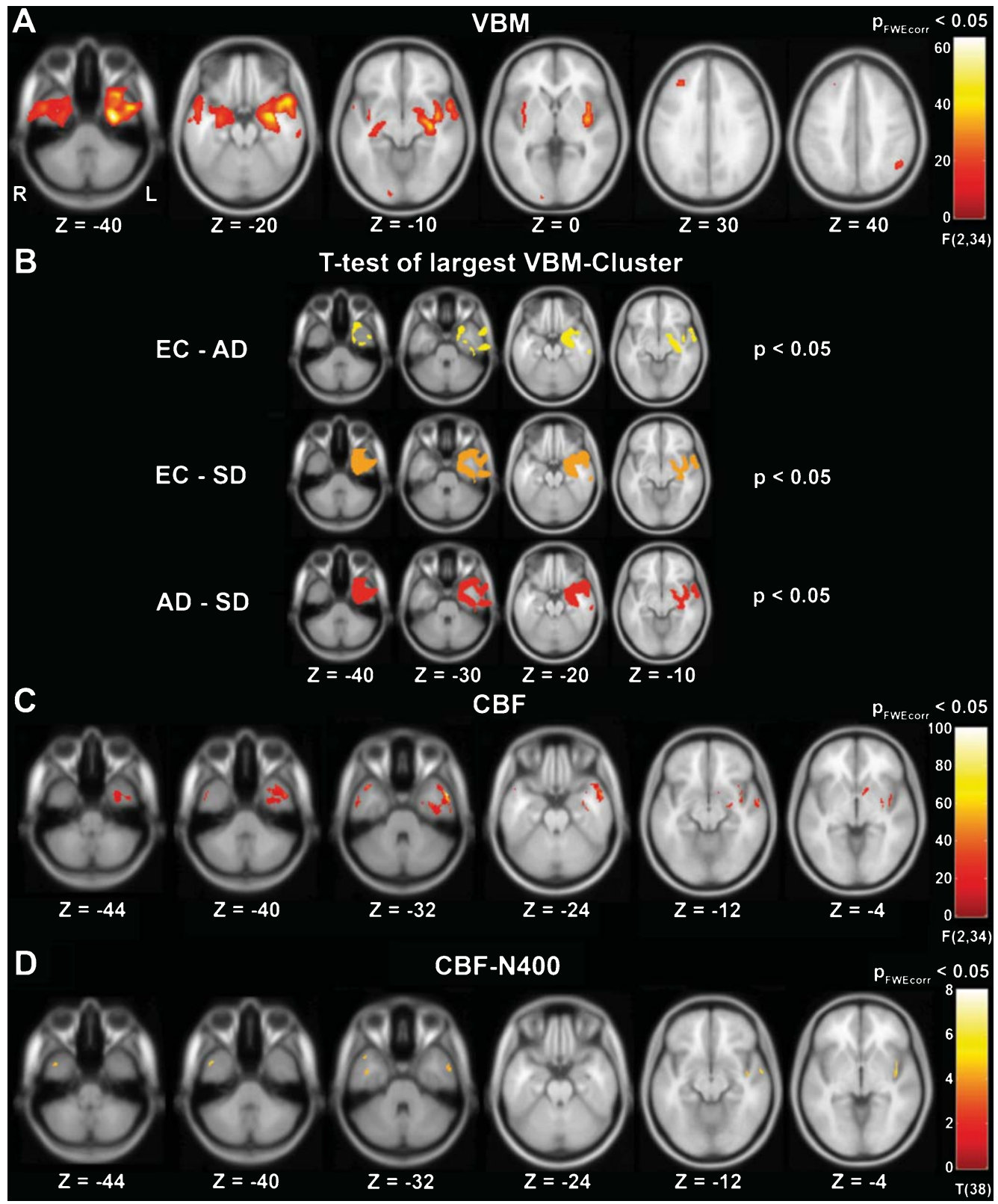

Fig. 5. A) Significant voxel-based morphometry (VBM) of group main-effect clusters on transverse slices of a standard average T1-image. B) Voxel-wise $T$-tests of the largest VBM cluster in the left temporal lobe involving 7,180 voxels. C) Significant cerebral blood flow (CBF) analysis of group main-effect clusters. D) Significant CBF-N400 linear regression clusters. AD, Alzheimer's disease; EC, elderly controls; SD, semantic dementia.

decreased CBF in the left insula, while only the SD group's $\mathrm{CBF}$ was lower in the left putamen and only the AD group's CBF was lower in the left globus pallidus. On the contralateral side, considerably smaller clusters that involved only the right middle temporal pole and the inferior temporal gyrus were found. In the former cluster, only the SD group differed from the EC group, while, in the latter cluster, both the AD and SD groups exhibited decreased $\mathrm{CBF}$.

\section{Linear regression $C B F-E R P$}

First, the linear regression of P1 and N1 yielded no significant clusters. Therefore, only results referring 
Table 4

Cerebral blood flow analysis of the anatomical regions that correspond to the MNI coordinates at the $F$ peak, the number of involved voxels and the peak $F$ and $p_{F W E-c o r r}$ values. Therefore, other brain structures were involved in the clusters, especially in the large ones (see text). Additionally, the post-hoc $T$-test results are listed with $T$ and $p$ values

\begin{tabular}{|c|c|c|c|c|c|c|c|c|}
\hline Anatomical region & $\mathrm{X}$ & $\mathrm{Y}$ & $\mathrm{Z}$ & \# of voxels & $F\left(p_{F W E-c o r r}\right)$ & EC-AD $T(p)$ & EC-SD $T(p)$ & AD-SD $T(p)$ \\
\hline $\mathrm{L}$ middle temporal pole & -54 & 6 & -32 & 1015 & $122.68\left(<0.001^{* * *}\right)$ & $3.86(<0.01 *)$ & $13.54(<0.001 * *)$ & $9.92(<0.001 * *)$ \\
\hline $\mathrm{R}$ middle temporal pole & 42 & 16 & -32 & 51 & $47.49(<0.001 * * *)$ & $2.83(0.009)$ & $6.81(<0.01 *)$ & $4.66(<0.01 *)$ \\
\hline $\mathrm{L}$ insula & -36 & -10 & -10 & 111 & $46.71(<0.001 * * *)$ & $3.65(<0.01 *)$ & $4.85(<0.01 *)$ & $3.37(0.02)$ \\
\hline L putamen & -32 & -12 & -4 & 57 & $39.30(<0.001 * * *)$ & $2.98(0.007)$ & $7.18(<0.01 *)$ & $4.79(<0.01 *)$ \\
\hline L globus pallidus & -12 & 6 & -6 & 64 & $32.51(<0.01 * *)$ & $4.72(<0.001 * *)$ & $3.40(0.02)$ & $2.09(0.1)$ \\
\hline $\mathrm{R}$ inferior temporal gyrus & 58 & -4 & -30 & 66 & $27.22\left(<0.05^{*}\right)$ & $3.60(<0.01 *)$ & $7.24(<0.01 *)$ & $4.14(<0.01 *)$ \\
\hline $\mathrm{L}$ inferior temporal gyrus & -60 & -18 & -28 & 21 & $27.19\left(<0.05^{*}\right)$ & $4.26(<0.001 * *)$ & $16.33(<0.001 * *)$ & $6.51(<0.001 * *)$ \\
\hline
\end{tabular}

T-test crit. $p$-value: $p<0.007$; $\mathrm{L}$ left; $\mathrm{R}$, right.

Table 5

CBF-Event-related potential linear regression of the anatomical regions and the $\mathrm{x}-\mathrm{y}-\mathrm{z}$ coordinates that correspond to the MNI coordinates at the $T$ peak, the number of involved voxels and the peak $T$ and $p_{F W E-c o r r}$ values

\begin{tabular}{|c|c|c|c|c|c|c|}
\hline Anatomical region & $X$ & $\mathrm{Y}$ & $\mathrm{Z}$ & \# of voxels & $T$ & $p_{F W E-c o r r}$ \\
\hline $\mathrm{R}$ middle temporal gyrus & 44 & -2 & -30 & 21 & 5.61 & $<0.01 * *$ \\
\hline $\mathrm{R}$ inferior temporal gyrus & 46 & 8 & -40 & 28 & 5.60 & $<0.001 * * *$ \\
\hline L insula & -42 & -2 & -6 & 70 & 5.58 & $<0.001 * * *$ \\
\hline L superior temporal gyrus & -60 & -4 & -12 & 42 & 5.24 & $<0.001 * * *$ \\
\hline L superior temporal pole & -32 & 12 & -28 & 13 & 5.09 & $<0.05^{*}$ \\
\hline L middle temporal pole & -38 & 6 & -36 & 36 & 4.82 & $<0.001 * * *$ \\
\hline $\mathrm{L}$ inferior temporal gyrus & -56 & 0 & -32 & 24 & 4.49 & $<0.01 * *$ \\
\hline
\end{tabular}

L, left; R, right.

to the linear regression of the N400 are reported. As such, seven clusters met the significance criteria of $p<0.0001$, as well as the FWE correction $(p<0.05)$, at the cluster level (Table 5). The clusters mainly converged with those of the voxel-based CBF statistics, except for those in the left superior temporal gyrus (Fig. 5D). However, the left putamen and globus pallidus did not show any significant relationship between $\mathrm{CBF}$ and the N400. The individual CBF and N400 correlation values can be drawn from the scatter plots of each cluster (Fig. 6). While the SD group exhibited lower values in both domains, the EC group showed the opposite results and the values in the $\mathrm{AD}$ group were intermediate. This observation raised the question of whether the $\mathrm{N} 400$ correlation can be regarded as a putative marker for dementia. For this purpose, the sensitivity, as well as the specificity, of separating the participant groups was calculated. First, the 95\% confidence intervals (CI) of the N400 correlation coefficients were derived for each group (CI for $\mathrm{EC}=0.48-0.74 ; \mathrm{AD}=0.05-0.45 ; \mathrm{SD}=-0.28-0.26$ ). Thus, the CIs of the EC and the AD groups did not overlap. Therefore, the coefficients between 0.45 and 0.48 reflected the boundary between healthy participants and demented ones. Such a separation was not possible between the AD and SD groups. Consequently, each individual's N400 correlation coefficient was compared with the cut-off values from 0.45 to 0.48 in the analysis (true positives $=15$; false negatives $=4$; true negatives $=15$; false positives $=4$ ). These results yielded a sensitivity and specificity of 0.79 each for all of the values between the CIs of the EC and AD groups because none of the participants had a N400 coefficient between 0.45 and 0.48 . Taken together, these results showed that decreased N400 similarity can be considered an indication of dementia. In addition, the N400 similarity was related to reduced $\mathrm{CBF}$ in the circumscribed brain areas, demonstrating an association of the two neurophysiological measures. However, according to the present data, a distinction between the AD and SD groups was not feasible by the decrease in N400 similarity.

\section{Correlations of the physiological parameters with the behavioral variables}

Table 6 displays all of the correlation indices between the physiological and the neuropsychological test parameters. The most important result of this analysis was the significantly positive correlation of the N400 similarity with the MMSE score and especially with the semantic test scores for the BNT, AF, and VF. In addition, all four of these tests were positively correlated, as expected. The moderate positive correlation 
R middle temporal gyrus

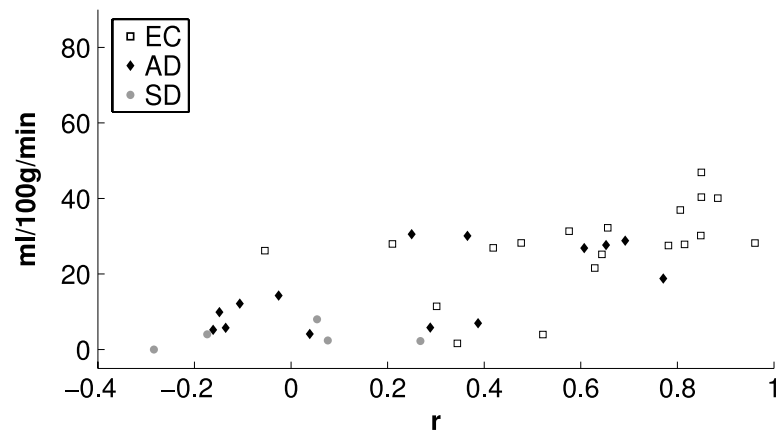

$L$ insula

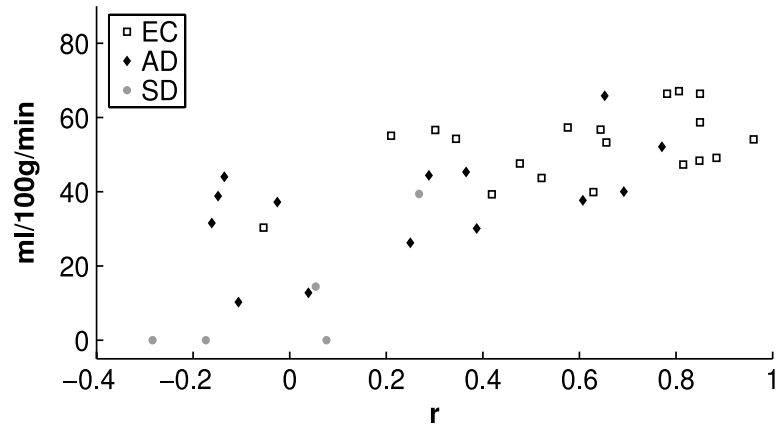

L superior temporal pole

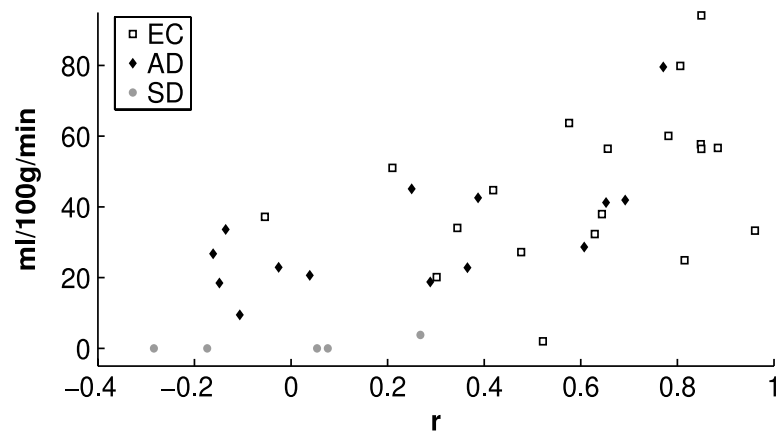

R inferior temporal gyrus

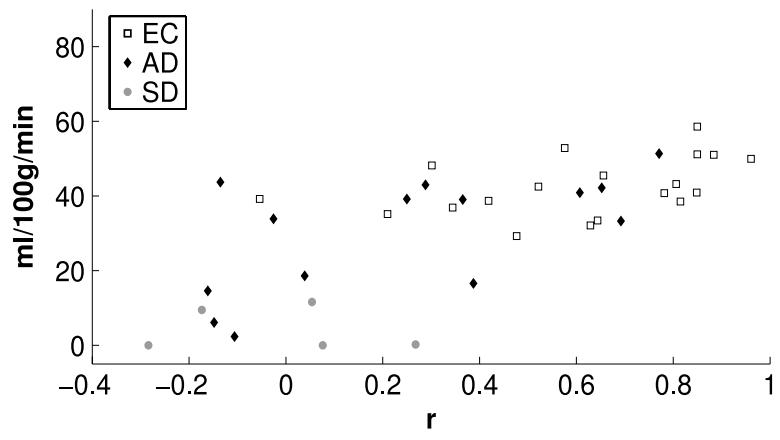

L superior temporal gyrus

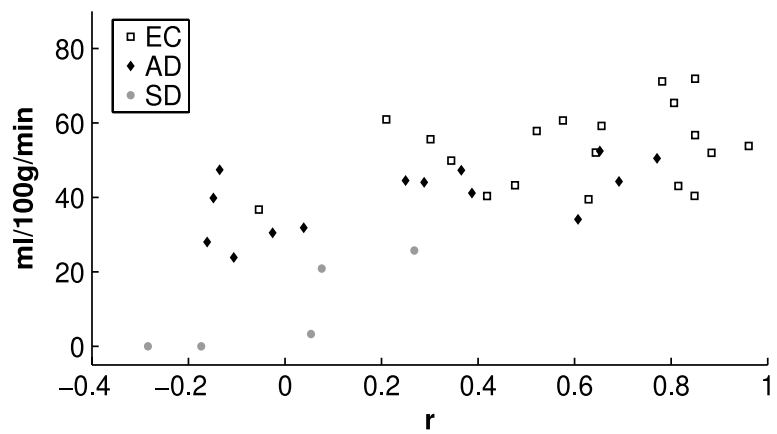

L middle temporal pole

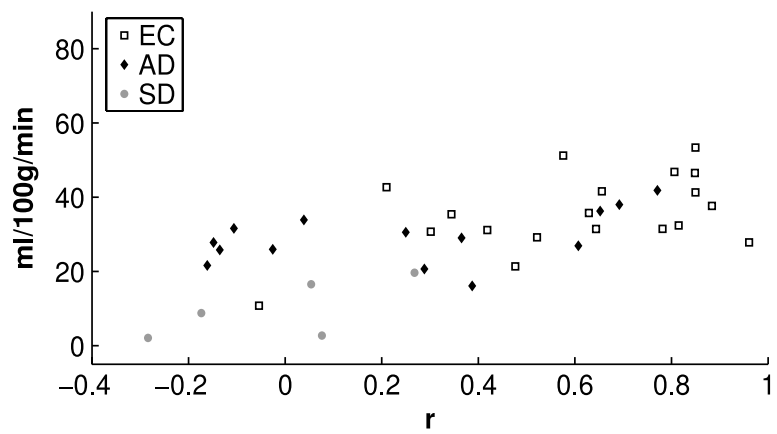

L inferior temporal gyrus

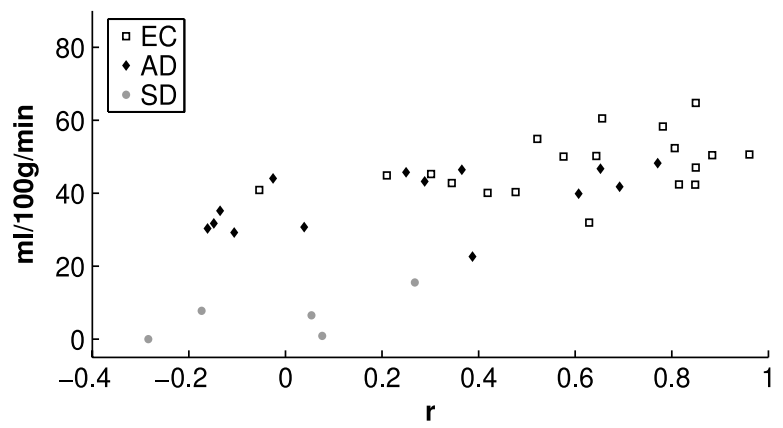

Fig. 6. Scatter plots for each cluster depicting individual N400 correlation (r) and cerebral blood blow. AD, Alzheimer's disease; EC, elderly controls; SD, semantic dementia. 
of the fluency scores with the CT construction might reflect that these tests required executive functions for successful performance.

\section{DISCUSSION}

The results of the present study indicated that the N400 topography might be a measure that should be further investigated in order to identify a cost-efficient and non-invasive marker of dementia. Moreover, a correlation of the N400 similarity with decreased $\mathrm{CBF}$ in the anterior temporal lobes was found, and this reflected the underlying pathology. Specifically, the combination of altered electrophysiology (N400), which was closely related to the patients' symptomatology as indicated by the positive correlations of the N400 similarity with the neuropsychological tests assessing semantic memory functions, with the neurophysiological aberrations (altered regional CBF) might help to further disentangle the pathological semantic memory processes in dementia. Before the crucial results involving the correlation of CBF and ERPs are discussed, other important findings in the voxel-based analyses, behavior, and ERPs are reviewed.

\section{Voxel-based analyses (VBM and CBF)}

The GM volume differences in the $\mathrm{AD}$ and $\mathrm{SD}$ groups compared to the EC group were mostly in line with previous findings [39-42, 44]. Besides the structural findings, the voxel-based CBF results showed that the anterior temporal lobes, in particular, were functionally altered in the $\mathrm{AD}$ and SD groups, as shown by decreased CBF. When comparing the number of significant voxels between each hemisphere, a moderate lateralization to the left can be observed. This applied primarily to the $\mathrm{AD}$ group, in which lowered $\mathrm{CBF}$ was found only in the left temporal poles, while lowered CBF was found bilaterally in the SD group. Taken together, for the $\mathrm{AD}$ group, these findings converge with those of earlier studies that investigated CBF changes $[48,50]$. Conversely, no hypermetabolism was measured in the medial temporal lobes in the $\mathrm{AD}$ group, which differed from the findings of Alsop et al. [49]. One reason for this divergence could be the milder $\mathrm{AD}$ condition of the current group (MMSE $=24.8$ ) compared to the group examined in Alsop's study (MMSE = 22.2). Nevertheless, this is the first study that investigated (ASL) CBF at rest in patients with SD. Despite the lack of comparable studies, the resulting brain areas that exhibited reduced CBF were in line with the assumptions. For instance, they concurred with Diehl's [52] findings about the decreased glucose metabolism in patients with SD.

\section{Behavior}

Besides the neurophysiological measures, the patients' symptomatology is of importance, because it can be understood as the behavioral manifestation of the above-mentioned structural and functional cerebral aberrations. In the present study, the AD and SD groups were comprised of individuals in an early dementia stage (see MMSE scores). However, both the BNT as well as the VF/AF showed that they suffered from severe semantic word retrieval deficits. Yet most of the patients were able to perform the LD task and showed semantic priming, as shown by the shorter RTs for related word pairs compared to unrelated word pairs. This was in accordance with the findings of Rogers et al. [22], who showed associative semantic priming both in the $\mathrm{AD}$ and $\mathrm{SD}$ groups. Thus, these results indicated that, in those patients, at least the automatic semantic processes (i.e., spread of activation) were still functional $[19,76]$.

\section{ERPs}

By first focusing on the waveforms (Fig. 3), one could observe that the waveforms of for example the F3 and Pz electrodes in the AD and EC groups converged with those found in the study of Revonsuo et al. [33]. Thus, the notion of the preserved spread of activation was supported and further substantiated by the comparable P1 and N1 topographies in the AD (and SD) groups in contrast to the EC template in this study, although the patients' correlation values were lower than those of the EC group. It was apparent that, for all ERPs, patients with SD exhibited the most deviant topographies, while those with AD showed intermediate topographic similarities that were between those of the EC and SD groups. The zero correlation of the SD group especially showed that there was no typical N400 topography present in this group, indicating severe deficits in controlled semantic word retrieval or a differing retrieval strategy. Thus, the current results indicated that the N400 separated the SD group from the AD and EC groups. However, the sensitivity and specificity analysis did not fully support this. Instead, it was found that the N400 correlation coefficient separated the healthy elderly from the patients with early dementia with a sensitivity and specificity of 0.79 . 
Table 6

Spearman rank-correlations $(\rho)$ of the neuropsychological measures with CBF and ERP variables

\begin{tabular}{|c|c|c|c|c|c|c|c|c|c|}
\hline & BNT & $\mathrm{AF}$ & VF & CTc & $\mathrm{CTr}$ & r-N400 & $\mathrm{r}-\mathrm{P} 1$ & $\mathrm{r}-\mathrm{N} 1$ & $\mathrm{CBF}$ \\
\hline MMSE & $0.56^{* *}$ & $0.63^{* *}$ & $0.66^{* *}$ & 0.30 & 0.22 & $0.54 * *$ & 0.17 & 0.23 & $0.32 *$ \\
\hline BNT & & $0.72^{* *}$ & $0.65^{* *}$ & 0.27 & 0.02 & $0.56 * *$ & 0.27 & 0.26 & 0.27 \\
\hline $\mathrm{AF}$ & & & $0.68 * *$ & $0.37 *$ & 0.19 & $0.47 * *$ & 0.14 & 0.32 & 0.31 \\
\hline VF & & & & $0.34 *$ & 0.26 & $0.53 * *$ & 0.20 & $0.33^{*}$ & $0.45^{* *}$ \\
\hline $\mathrm{CTc}$ & & & & & $0.34 *$ & 0.05 & 0.15 & 0.15 & 0.10 \\
\hline $\mathrm{CTr}$ & & & & & & 0.07 & 0.01 & 0.18 & $0.34 *$ \\
\hline r-N400 & & & & & & & 0.20 & 0.20 & $0.36^{*}$ \\
\hline r-P1 & & & & & & & & $0.38 *$ & 0.04 \\
\hline $\mathrm{r}-\mathrm{N} 1$ & & & & & & & & & 0.31 \\
\hline
\end{tabular}

$* p<0.05, * * p<0.01$. MMSE, Mini-Mental State Examination; BNT, Boston Naming Test; AF, animal fluency; VF, verb fluency; CTc, clock task construction; CTr, clock task reading; r-N400, N400 similarity; r-P1, P1 similarity; r-N1, N1 similarity; CBF, global CBF grey matter masked.

Hence, this result revealed that the N400 similarity was a putative marker for early dementia but not for a differential diagnosis of $\mathrm{AD}$ or SD. Of course, the fact that this result was based on a small participant group size, larger samples and especially longitudinal studies are needed in order to verify the current finding. For instance, the possibility cannot be excluded that either the false-positive EC participants will later develop dementia or those with low N400 similarity have decreased controlled semantic memory abilities in general.

In summary, it was surprising that the SD group showed comparable early ERPs as the EC and AD groups because a loss of semantic knowledge would have also led to an aberrant spread of activation. Therefore, it is noteworthy that this is the first study to detect $\mathrm{P} 1$ and N1 of semantic word processing in the SD group. This result suggested the preserved automatic processes in patients with $\mathrm{AD}[77,78]$ and in patients with SD, which is an important finding for future studies and for clinical implications. Furthermore, when attempting to compare the current ERP findings to the existing literature, consistency was found in the altered N400 topography that was related to impaired semantic word retrieval in patients with AD and SD [29, 35, 37]. Nevertheless, the observation that the AD's N400 topography still had a typical shape might indicate preserved semantic knowledge but impaired access, and this indication converges with those of Ford et al. [34] and Auchterlonie and colleagues [35].

\section{Linear regression of $C B F$ and $E R P S$}

Contradicting the assumptions made in this study, neither the P1 nor the $\mathrm{N} 1$ topographies seemed to be related to changes in $\mathrm{CBF}$. One reason might have been that the automatic spread of activation in the semantic network was not functionally changed in the patients, as discussed above. Opposed to this, the preserved N400 topography was associated with higher $\mathrm{CBF}$, especially in the left temporal pole and the lateral temporal lobe, but also in the left insula and the right lateral temporal lobe. Congruous with the voxel-based results, the clusters in the temporal pole exclusively in the left hemisphere indicated a lateralization to the language-dominant hemisphere. Thus, the present results indicated that the controlled semantic word retrieval relied strongly on anterior temporal lobe functions. This view was supported by a study of Wirth et al. [25], which showed that the N400 is related to bilateral activation in the temporal lobes. Accordingly, the observations that the patients with SD showed severe GM atrophy in this region, dramatically reduced baseline blood flow and almost no N400 appeared consistent. Moreover, in patients with $\mathrm{AD}, \mathrm{GM}$ volume loss was less extended, as was their CBF reduction. Therefore, the controlled semantic word retrieval was preserved to some degree, as expressed by the rather typical N400 topography, although it was altered compared to that of the EC group. Taken together, the potential of the N400 topography as an early marker for dementia was supported by its relationship to the structural and functional neuropathology in dementia, as was demonstrated in the present study and in recent studies (e.g., [36, 79, 80]).

\section{CONCLUSION}

The findings of the present study contributed to the understanding of the underlying mechanisms of semantic memory deterioration in patients with $\mathrm{AD}$ and those with SD based on three modalities: structure (GM volume), baseline metabolism (rest CBF), and task functionality (ERPs). In particular, these results showed a large overlap of reduced blood flow in the 
brain areas that were affected by GM volume loss. Furthermore, deviant N400 topographies were associated with reduced $\mathrm{CBF}$ in most of these regions. Namely, the temporal lobes bilaterally, which trigger the N400 that is related to lexical-semantic integration $[25,81]$, appeared to play a crucial role in the interaction of atrophy, altered baseline blood flow, and impaired controlled semantic word retrieval.

Even though these results seem promising, the sole use of the N400 topography as an early marker for dementia is not yet applicable and needs replication in additional studies with independent participant groups. However, the high sensitivity of the N400 similarity may be a motivation for forthcoming studies to validate this marker in order to establish a cost-efficient and non-invasive diagnostic tool that complements those already available. To this end, future studies need to replicate and refine the current findings in order to support the use of the N400 for a differential diagnostic purpose. Moreover, with the prospect of improving the understanding and sensitivity of the ERPs, longitudinal studies are needed to elucidate the individual development of the early P1 and N1 and the late N400 topographies, ideally in the elderly who are healthy and those who convert to dementia. For instance, Bobes et al. [82] have reported that altered N400 topographies might occur even before the manifestation of semantic memory symptoms.

Other limitations of this study need to be considered. A major problem was the small group sizes, especially in the SD group. In particular, quite a few analyses showed tendencies to statistical significance, suggesting that more effects might have emerged with larger sample sizes. As such, a generalization of the present findings appeared difficult. Nevertheless, because SD is a relatively rare condition in the general population and is often not diagnosed, this sample was nevertheless of precious value. The fact that these five SD patients provided functional data in a task that was closely related to their cognitive deficits, as well as the structural and baseline blood flow images, makes this notion even stronger. Finally, it has to be examined whether the $\mathrm{N} 400$ similarity is specific for patients with dementia or is also found in patients with other disorders.

In conclusion, the present study demonstrated that the altered N400 electrophysiology in patients with dementia with semantic memory impairments was closely related to their structural and baseline blood flow degeneration, and this was found distinctly in regions that are involved in controlled semantic word processing.

\section{ACKNOWLEDGMENTS}

This study was supported by the Swiss Synapsis Foundation and the Swedish Alzheimerfonden. We thank the following contributors: Linnea Engström, Eric Westman and Olof Lindberg of the Karolinska Institute, Stockholm, Sweden for performing part of the neuropsychological testing; Francisco Lacerda and Petter Kallioinen of the Department of Linguistics, Stockholm University, Stockholm, Sweden for their invaluable support at the EEG lab; and Tie-Qiang Li of the Karolinska Institute, Stockholm, Sweden for his support during the MR measurements.

Authors' disclosures available online (http://www.jalz.com/disclosures/view.php?id=1757).

\section{REFERENCES}

[1] Castellani RJ, Rolston RK, Smith MA (2010) Alzheimer disease. Dis Mon 56, 484-546.

[2] Lopes MA, Hototian SR, Bustamante SE, Azevedo D, Tatsch M, Bazzarella MC, Litvoc J, Bottino CM (2007) Prevalence of cognitive and functional impairment in a community sample in Ribeirao Preto, Brazil. Int J Geriatr Psychiatry 22, 770-776.

[3] Fratiglioni L, De Ronchi D, Aguero-Torres H (1999) Worldwide prevalence and incidence of dementia. Drugs Aging 15, 365-375.

[4] Brugger P, Monsch AU, Salmon DP, Butters N (1996) Random number generation in dementia of the Alzheimer type: A test of frontal executive functions. Neuropsychologia 34, 97-103.

[5] Sebastian Gascon MV, Hernandez-Gil L (2010) A comparison of memory and executive functions in Alzheimer disease and the frontal variant of frontotemporal dementia. Psicothema 22, 424-429.

[6] Nyatsanza S, Shetty T, Gregory C, Lough S, Dawson K, Hodges JR (2003) A study of stereotypic behaviours in Alzheimer's disease and frontal and temporal variant frontotemporal dementia. J Neurol Neurosurg Psychiatry 74, 1398-1402.

[7] Hodges JR (2001) Frontotemporal dementia (Pick's disease): Clinical features and assessment. Neurology 56, S6-10.

[8] Snowden JS, Neary D, Mann DM, Goulding PJ, Testa HJ (1992) Progressive language disorder due to lobar atrophy. Ann Neurol 31, 174-183.

[9] Gorno-Tempini ML, Hillis AE, Weintraub S, Kertesz A, Mendez M, Cappa SF, Ogar JM, Rohrer JD, Black S, Boeve BF, Manes F, Dronkers NF, Vandenberghe R, Rascovsky K, Patterson K, Miller BL, Knopman DS, Hodges JR, Mesulam MM, Grossman M (2011) Classification of primary progressive aphasia and its variants. Neurology 76, 1006-1014.

[10] Jack CR, Albert MS, Knopman DS, McKhann GM, Sperling RA, Carrillo MC, Thies B, Phelps CH (2011) Introduction to the recommendations from the National Institute on Aging-Alzheimer's Association workgroups on diagnostic guidelines for Alzheimer's disease. Alzheimers Dement 7, 257-262.

[11] Perrin RJ, Fagan AM, Holtzman DM (2009) Multimodal techniques for diagnosis and prognosis of Alzheimer's disease. Nature 461, 916-922. 
[12] Neary D, Snowden JS, Gustafson L, Passant U, Stuss D, Black S, Freedman M, Kertesz A, Robert PH, Albert M, Boone K, Miller BL, Cummings J, Benson DF (1998) Frontotemporal lobar degeneration: A consensus on clinical diagnostic criteria. Neurology 51, 1546-1554.

[13] Pengas G, Patterson K, Arnold RJ, Bird CM, Burgess N, Nestor PJ (2010) Lost and found: Bespoke memory testing for Alzheimer's disease and semantic dementia. J Alzheimers Dis 21, 1347-1365.

[14] Austin BP, Nair VA, Meier TB, Xu G, Rowley HA, Carlsson CM, Johnson SC, Prabhakaran V (2011) Effects of hypoperfusion in Alzheimer's disease. J Alzheimers Dis 26(Suppl 3), 123-133.

[15] Sperling RA, Aisen PS, Beckett LA, Bennett DA, Craft S, Fagan AM, Iwatsubo T, Jack CR Jr, Kaye J, Montine TJ, Park DC, Reiman EM, Rowe CC, Siemers E, Stern Y, Yaffe K, Carrillo MC, Thies B, Morrison-Bogorad M, Wagster MV, Phelps CH (2011) Toward defining the preclinical stages of Alzheimer's disease: Recommendations from the National Institute on Aging-Alzheimer's Association workgroups on diagnostic guidelines for Alzheimer's disease. Alzheimers Dement 7, 280-292.

[16] Blackwell AD, Sahakian BJ, Vesey R, Semple JM, Robbins TW, Hodges JR (2004) Detecting dementia: Novel neuropsychological markers of preclinical Alzheimer's disease. Dement Geriatr Cogn Disord 17, 42-48.

[17] Vogel A, Gade A, Stokholm J, Waldemar G (2005) Semantic memory impairment in the earliest phases of Alzheimer's disease. Dement Geriatr Cogn Disord 19, 75-81.

[18] Ober BA (2002) RT and non-RT methodology for semantic priming research with Alzheimer's disease patients: A critical review. J Clin Exp Neuropsychol 24, 883-911.

[19] Giffard B, Laisney M, Mezenge F, de la Sayette V, Eustache F, Desgranges B (2008) The neural substrates of semantic memory deficits in early Alzheimer's disease: Clues from semantic priming effects and FDG-PET. Neuropsychologia 46, 1657-1666.

[20] Ober BA, Shenaut GK (1988) Lexical decision and priming in Alzheimer's disease. Neuropsychologia 26, 273-286.

[21] Nebes RD, Martin DC, Horn LC (1984) Sparing of semantic memory in Alzheimer's disease. J Abnorm Psychol 93, 321330.

[22] Rogers SL, Friedman RB (2008) The underlying mechanisms of semantic memory loss in Alzheimer's disease and semantic dementia. Neuropsychologia 46, 12-21.

[23] Chertkow H, Bub D, Bergman H, Bruemmer A, Merling A, Rothfleisch J (1994) Increased semantic priming in patients with dementia of the Alzheimer's type. J Clin Exp Neuropsychol 16, 608-622.

[24] Grieder M, Crinelli RM, Koenig T, Wahlund LO, Dierks T, Wirth M (2012) Electrophysiological and behavioral correlates of stable automatic semantic retrieval in aging. Neuropsychologia 50, 160-171.

[25] Wirth M, Horn H, Koenig T, Stein M, Federspiel A, Meier B, Michel CM, Strik W (2007) Sex differences in semantic processing: Event-related brain potentials distinguish between lower and higher order semantic analysis during word reading. Cereb Cortex 17, 1987-1997.

[26] Kutas M, Federmeier KD (2000) Electrophysiology reveals semantic memory use in language comprehension. Trends Cogn Sci 4, 463-470.

[27] Khateb A, Pegna AJ, Landis T, Mouthon MS, Annoni JM (2010) On the origin of the N400 effects: An ERP waveform and source localization analysis in three matching tasks. Brain Topogr 23, 311-320.
[28] Taylor JR, Olichney JM (2007) From amnesia to dementia: ERP studies of memory and language. Clin EEG Neurosci 38, 8-17.

[29] Schwartz TJ, Kutas M, Butters N, Paulsen JS, Salmon DP (1996) Electrophysiological insights into the nature of the semantic deficit in Alzheimer's disease. Neuropsychologia 34, 827-841.

[30] Iragui V, Kutas M, Salmon DP (1996) Event-related brain potentials during semantic categorization in normal aging and senile dementia of the Alzheimer's type. Electroencephalogr Clin Neurophysiol 100, 392-406.

[31] Ford JM, Woodward SH, Sullivan EV, Isaacks BG, Tinklenberg JR, Yesavage JA, Roth WT (1996) N400 evidence of abnormal responses to speech in Alzheimer's disease. Electroencephalogr Clin Neurophysiol 99, 235-246.

[32] Hurley RS, Paller KA, Rogalski EJ, Mesulam MM (2012) Neural mechanisms of object naming and word comprehension in primary progressive aphasia. J Neurosci 32, 4848-4855.

[33] Revonsuo A, Portin R, Juottonen K, Rinne JO (1998) Semantic processing of spoken words in Alzheimer's disease: An electrophysiological study. J Cogn Neurosci 10, 408-420.

[34] Ford JM, Askari N, Mathalon DH, Menon V, Gabrieli JD, Tinklenberg JR, Yesavage J (2001) Event-related brain potential evidence of spared knowledge in Alzheimer's disease. Psychol Aging 16, 161-176.

[35] Auchterlonie S, Phillips NA, Chertkow H (2002) Behavioral and electrical brain measures of semantic priming in patients with Alzheimer's disease: Implications for access failure versus deterioration hypotheses. Brain Cogn 48, 264-267.

[36] Olichney JM, Yang JC, Taylor J, Kutas M (2011) Cognitive event-related potentials: Biomarkers of synaptic dysfunction across the stages of Alzheimer's disease. J Alzheimers Dis 26(Suppl 3), 215-228.

[37] Olichney JM, Taylor JR, Gatherwright J, Salmon DP, Bressler AJ, Kutas M, Iragui-Madoz VJ (2008) Patients with MCI and $\mathrm{N} 400$ or P600 abnormalities are at very high risk for conversion to dementia. Neurology 70, 1763-1770.

[38] Hamberger MJ, Friedman D, Ritter W, Rosen J (1995) Event-related potential and behavioral correlates of semantic processing in Alzheimer's patients and normal controls. Brain Lang 48, 33-68.

[39] Boxer AL, Rankin KP, Miller BL, Schuff N, Weiner M, Gorno-Tempini ML, Rosen HJ (2003) Cinguloparietal atrophy distinguishes Alzheimer disease from semantic dementia. Arch Neurol 60, 949-956.

[40] Galton CJ, Patterson K, Graham K, Lambon-Ralph MA, Williams G, Antoun N, Sahakian BJ, Hodges JR (2001) Differing patterns of temporal atrophy in Alzheimer's disease and semantic dementia. Neurology 57, 216-225.

[41] Rami L, Gomez-Anson B, Monte GC, Bosch B, SanchezValle R, Molinuevo JL (2009) Voxel based morphometry features and follow-up of amnestic patients at high risk for Alzheimer's disease conversion. Int J Geriatr Psychiatry 24, 875-884.

[42] Mummery CJ, Patterson K, Price CJ, Ashburner J, Frackowiak RS, Hodges JR (2000) A voxel-based morphometry study of semantic dementia: Relationship between temporal lobe atrophy and semantic memory. Ann Neurol 47, 36-45.

[43] Rosen HJ, Gorno-Tempini ML, Goldman WP, Perry RJ, Schuff N, Weiner M, Feiwell R, Kramer JH, Miller BL (2002) Patterns of brain atrophy in frontotemporal dementia and semantic dementia. Neurology 58, 198-208.

[44] Frings L, Mader I, Landwehrmeyer BG, Weiller C, Hull M, Huppertz HJ (2012) Quantifying change in individual subjects 
affected by frontotemporal lobar degeneration using automated longitudinal MRI volumetry. Hum Brain Mapp 33, 1526-1535.

[45] Luckhaus C, Janner M, Cohnen M, Fluss MO, Teipel SJ, Grothe M, Hampel H, Kornhuber J, Ruther E, Peters O, Supprian T, Gaebel W, Modder U, Wittsack HJ (2010) A novel MRI-biomarker candidate for Alzheimer's disease composed of regional brain volume and perfusion variables. Eur J Neurol 17, 1437-1444.

[46] Alsop DC, Dai W, Grossman M, Detre JA (2010) Arterial spin labeling blood flow MRI: Its role in the early characterization of Alzheimer's disease. J Alzheimers Dis 20, 871880.

[47] Chen Y, Wolk DA, Reddin JS, Korczykowski M, Martinez PM, Musiek ES, Newberg AB, Julin P, Arnold SE, Greenberg JH, Detre JA (2011) Voxel-level comparison of arterial spinlabeled perfusion MRI and FDG-PET in Alzheimer disease. Neurology 77, 1977-1985.

[48] Johnson NA, Jahng GH, Weiner MW, Miller BL, Chui HC, Jagust WJ, Gorno-Tempini ML, Schuff N (2005) Pattern of cerebral hypoperfusion in Alzheimer disease and mild cognitive impairment measured with arterial spinlabeling MR imaging: Initial experience. Radiology 234, 851859.

[49] Alsop DC, Casement M, de Bazelaire C, Fong T, Press DZ (2008) Hippocampal hyperperfusion in Alzheimer's disease. Neuroimage 42, 1267-1274.

[50] Dai W, Lopez OL, Carmichael OT, Becker JT, Kuller LH, Gach HM (2009) Mild cognitive impairment and alzheimer disease: Patterns of altered cerebral blood flow at MR imaging. Radiology 250, 856-866.

[51] Fleisher AS, Podraza KM, Bangen KJ, Taylor C, Sherzai A, Sidhar K, Liu TT, Dale AM, Buxton RB (2009) Cerebral perfusion and oxygenation differences in Alzheimer's disease risk. Neurobiol Aging 30, 1737-1748.

[52] Diehl J, Grimmer T, Drzezga A, Riemenschneider M, Forstl H, Kurz A (2004) Cerebral metabolic patterns at early stages of frontotemporal dementia and semantic dementia. A PET study. Neurobiol Aging 25, 1051-1056.

[53] Michel CM, Seeck M, Murray MM (2004) The speed of visual cognition. Suppl Clin Neurophysiol 57, 617-627.

[54] Halgren E, Baudena P, Heit G, Clarke JM, Marinkovic K, Clarke M (1994) Spatio-temporal stages in face and word processing. I. Depth-recorded potentials in the human occipital, temporal and parietal lobes [corrected]. J Physiol Paris 88, $1-50$.

[55] Halgren E, Dhond RP, Christensen N, Van Petten C, Marinkovic K, Lewine JD, Dale AM (2002) N400-like magnetoencephalography responses modulated by semantic context, word frequency, and lexical class in sentences. $\mathrm{Neu}$ roimage $17,1101-1116$.

[56] The ICD-10 Classification of Mental and Behavioral Disorders (1992) World Health Organization Geneva.

[57] Haatveit BC, Sundet K, Hugdahl K, Ueland T, Melle I, Andreassen OA (2010) The validity of d prime as a working memory index: Results from the "Bergen n-back task". $J$ Clin Exp Neuropsychol 32, 871-880.

[58] Tran Y, Craig A, Boord P, Craig D (2004) Using independent component analysis to remove artifact from electroencephalographic measured during stuttered speech. Med Biol Eng Comput 42, 627-633.

[59] Lehmann D, Skrandies W (1980) Reference-free identification of components of checkerboard-evoked multichannel potential fields. Electroencephalogr Clin Neurophysiol 48, 609-621.
[60] Murray MM, Brunet D, Michel CM (2008) Topographic ERP analyses: A step-by-step tutorial review. Brain Topogr 20, 249-264.

[61] Koenig T, Kottlow M, Stein M, Melie-Garcia L (2011) Ragu: A free tool for the analysis of EEG and MEG event-related scalp field data using global randomization statistics. Comput Intell Neurosci 2011, 938925.

[62] Koenig T, Melie-Garcia L (2009) Statistical analysis of multichannel scalp field data. In Electrical Neuroimaging, Michel CM, Koenig T, Brandeis D, Gianotti LRR, Wackermann J, eds. Cambridge University Press, New York, pp. 169-190.

[63] Pascual-Marqui RD, Michel CM, Lehmann D (1995) Segmentation of brain electrical activity into microstates: Model estimation and validation. IEEE Trans Biomed Eng 42, 658665.

[64] Brandeis D, Naylor H, Halliday R, Callaway E, Yano L (1992) Scopolamine effects on visual information processing, attention, and event-related potential map latencies. Psychophysiology 29, 315-336.

[65] Wu WC, Fernandez-Seara M, Detre JA, Wehrli FW, Wang J (2007) A theoretical and experimental investigation of the tagging efficiency of pseudocontinuous arterial spin labeling. Magn Reson Med 58, 1020-1027.

[66] Dai W, Garcia D, de Bazelaire C, Alsop DC (2008) Continuous flow-driven inversion for arterial spin labeling using pulsed radio frequency and gradient fields. Magn Reson Med 60, 1488-1497.

[67] Ashburner J, Friston KJ (2000) Voxel-based morphometrythe methods. Neuroimage 11, 805-821.

[68] Good CD, Johnsrude IS, Ashburner J, Henson RN, Friston KJ, Frackowiak RS (2001) A voxel-based morphometric study of ageing in 465 normal adult human brains. Neuroimage 14, 21-36.

[69] Wang J, Alsop DC, Song HK, Maldjian JA, Tang K, Salvucci AE, Detre JA (2003) Arterial transit time imaging with flow encoding arterial spin tagging (FEAST). Magn Reson Med 50, 599-607.

[70] Federspiel A, Muller TJ, Horn H, Kiefer C, Strik WK (2006) Comparison of spatial and temporal pattern for fMRI obtained with BOLD and arterial spin labeling. J Neural Transm 113, 1403-1415.

[71] Luh WM, Wong EC, Bandettini PA, Hyde JS (1999) QUIPSS II with thin-slice TI1 periodic saturation: A method for improving accuracy of quantitative perfusion imaging using pulsed arterial spin labeling. Magn Reson Med 41, 12461254.

[72] Wang J, Wang Z, Aguirre GK, Detre JA (2005) To smooth or not to smooth? ROC analysis of perfusion fMRI data. Magn Reson Imaging 23, 75-81.

[73] Focke NK, Helms G, Kaspar S, Diederich C, Toth V, Dechent P, Mohr A, Paulus W (2011) Multi-site voxel-based morphometry-not quite there yet. Neuroimage 56, 11641170 .

[74] Jann K, Koenig T, Dierks T, Boesch C, Federspiel A (2010) Association of individual resting state EEG alpha frequency and cerebral blood flow. Neuroimage 51, 365-372.

[75] Pulvermuller F, Assadollahi R, Elbert T (2001) Neuromagnetic evidence for early semantic access in word recognition. Eur J Neurosci 13, 201-205.

[76] Hill H, Strube M, Roesch-Ely D, Weisbrod M (2002) Automatic vs. controlled processes in semantic primingdifferentiation by event-related potentials. Int J Psychophysiol 44, 197-218.

[77] Ober BA, Shenaut GK, Jagust WJ, Stillman RC (1991) Automatic semantic priming with various category relations in 
Alzheimer's disease and normal aging. Psychol Aging 6, 647660 .

[78] Nebes RD (1989) Semantic memory in Alzheimer's disease. Psychol Bull 106, 377-394.

[79] Fennema-Notestine C, Hagler DJ, Jr., McEvoy LK, Fleisher AS, Wu EH, Karow DS, Dale AM, Alzheimer's Disease Neuroimaging, Initiative (2009) Structural MRI biomarkers for preclinical and mild Alzheimer's disease. Hum Brain Mapp 30, 3238-3253.

[80] Peters F, Majerus S, Collette F, Degueldre C, Del Fiore G, Laureys S, Moonen G, Salmon E (2009) Neural sub- strates of phonological and lexicosemantic representations in Alzheimer's disease. Hum Brain Mapp 30, 185-199.

[81] McCarthy G, Nobre AC, Bentin S, Spencer DD (1995) Language-related field potentials in the anterior-medial temporal lobe: I. Intracranial distribution and neural generators. J Neurosci 15, 1080-1089.

[82] Bobes MA, Garcia YF, Lopera F, Quiroz YT, Galan L, Vega M, Trujillo N, Valdes-Sosa M, Valdes-Sosa P (2010) ERP generator anomalies in presymptomatic carriers of the Alzheimer's disease E280A PS-1 mutation. Hum Brain Mapp 31, 247-265. 